\title{
From Diophantus to supergravity and massless higher spin multiplets
}

\section{S. James Gates Jr. ${ }^{a, b}$ and Konstantinos Koutrolikos ${ }^{c}$}

\author{
${ }^{a}$ Center for String and Particle Theory-Dept. of Physics, University of Maryland, \\ 4150 Campus Dr., College Park, MD 20472, U.S.A. \\ ${ }^{b}$ Department of Physics, Brown University, \\ Box 1843, 182 Hope Street, Barus 6 Holley 545, Providence, RI 02912, U.S.A. \\ ${ }^{c}$ Institute for Theoretical Physics and Astrophysics, Masaryk University, \\ 61137 Brno, Czech Republic \\ E-mail: gatess@wam.umd.edu, kkoutrolikos@physics.muni.cz
}

ABSTRACT: We present a new method of deriving the off-shell spectrum of supergravity and massless $4 D, \mathcal{N}=1$ higher spin multiplets without the need of an action and based on a set of natural requirements: (a.) existence of an underlying superspace description, (b.) an economical description of free, massless, higher spins and (c.) equal numbers of bosonic and fermionic degrees of freedom. We prove that for any theory that respects the above, the fermionic auxiliary components come in pairs and are gauge invariant and there are two types of bosonic auxiliary components. Type (1) are pairs of a (2,0)-tensor with real or imaginary $(1,1)$-tensor with non-trivial gauge transformations. Type (2) are singlets and gauge invariant. The outcome is a set of Diophantine equations, the solutions of which determine the off-shell spectrum of supergravity and massless higher spin multiplets. This approach provides $(i)$ a classification of the irreducible, supersymmetric, representations of arbitrary spin and (ii) a very clean and intuitive explanation to why some of these theories have more than one formulations (e.g. the supergravity multiplet) and others do not.

KEYWords: Supergravity Models, Superspaces, Supersymmetric Effective Theories

ArXIV EPRINT: 1707.00194 


\section{Contents}

1 Introduction 1

2 On-shell data and the higher spin requirement 3

3 Demanding supersymmetry 4

3.1 Types of auxiliary fields 4

$\begin{array}{ll}3.2 & \text { Matching bosons with fermions }\end{array}$

4 Demanding existence of a superspace descriptions $\quad 8$

4.1 Types of superfields 8

4.2 Gauge transformations 9

5 Demanding economy $\quad 12$

$\begin{array}{lll}6 & Y=1 / 2-\text { vector supermultiplet } & 13\end{array}$

$7 Y=1-$ matter-gravitino supermultiplets $\quad 14$

$\begin{array}{lll}8 & Y=3 / 2 & 18\end{array}$

9 Arbitrary half-integer superspin $\quad 25$

10 Arbitrary integer superspin $\quad 29$

11 Discussion $\quad 32$

\section{Introduction}

The study of higher spin theories is very well motivated these days in part due to their increasing relevance originating from string theory and holography. In string theory, one can argue that many of the attractive UV properties of scattering amplitudes calculated perturbatively originate from the transmission of higher spin particles at the corresponding exchange. Also, there is the old conjecture that the massless phase of the theory emerging at some ultra-high energy regime (i.e. the "tensionaless string"), will be governed by some sort of a higher spin gauge theory. For holography, the appearance of higher spins is slightly more subtle. Interacting higher spin theories are very difficult to construct, but there has been some success and all of them have the same two features in common. The existence of a massless spin 2 state and a non-flat background (usually AdS). These two properties, make higher spins the perfect laboratory for investigating holography. 
In many of the above described theories, supersymmetry is a vital ingredient and therefore it is natural to ask for the description of higher spins in the presence of supersymmetry. Higher spin irreducible representations (irreps) of the supersymmetric extension of the Poincaré symmetry group have been studied in detail in 4D where the meaning of spin and mass is clear. On the one hand there is the, group theoretic, on-shell approach which provides the set of conditions we must impose on tensors in order to describe irreducible supermultiplets. This is very important because every theory must respect these conditions at the zero coupling limit and properly deform, without negating, them when interactions are restored. One of the well known results of this approach is the on-shell spectrum of the theory, which is related with the decomposition of the irreps of the super-Poincaré group to irreps of the Poincaré group. For example, massless, $\mathcal{N}=1$ supermultiplets of superspin $Y$ include one spin $j=Y$ and one spin $j=Y+1 / 2$. This is due to the fact that for massless theories only $1 / 4$ of the supersymmetry generators can be used for creation operators (ascending ladder operators) and each one can be used at most once. Therefore for $\mathcal{N}=1$, there is only one creation operator and therefore we have two states, the 'vacuum' $(j=Y)$ and its first excitation $(j=Y+1 / 2)$.

On the other hand, there is the off-shell description of the theory, where one is writing actions that provide equations of motion compatible with the previously mentioned on-shell conditions. This is the first step towards the fully interacting theory (if it exists). Such constructions exist for massless $\mathcal{N}=1$ theories of arbitrary high spin [1-3] but not for massive theories $[4,5]$. The main result of this approach is the off-shell spectrum of the theory, meaning the set of auxiliary components that supersymmetry introduces on top of the components required for the description of propagating higher spin fields. Although there has been a very detailed derivation [3] of the off-shell spectrum for massless theories, there is no intuitive argument that leads directly to these results.

The purpose of this paper is to fill this gap and provide an alternative derivation of the set of off-shell auxiliary components required by supersymmetry without having to (a.) find the free higher spin superspace action first and then (b.) project to components to extract the off-shell spectrum of the theory. We will show that there is a set of natural requirements that fix the auxiliary components completely for any spin and allow us to deduce the required gauge transformations that lead uniquely to the action and generate all the details. In other words, we present a method that given the on-shell spectrum of massless $4 D, \mathcal{N}=1$ theories (the spins we want to describe) produces the off-shell completion, i.e. the set of auxiliary fields required, their gauge transformations, the set of superfields that generate these fields and give the superspace description of the theory and their superspace gauge transformations. We hope this addition to the understanding of the structure of the auxiliary components of supersymmetric theories can eventually help towards finding the superspace description of massive higher spin systems and their future development.

The method relies on a list of requirements, whose imposition extracts the set of desired theories from the set of all possible theories. As we are interested in supersymmetric theories, one of the requirements must provide this information. We do this by demanding the equality of the bosonic degrees of freedom (d.o.f) of the theory with the fermionic d.o.f. 
This is a natural requirement that all supersymmetric theories must respect off-shell. For future reference we will call this the Supersymmetry requirement.

Furthermore, it is natural to have the property of supersymmetry manifest. This would mean that there must exist a superspace description of the theory, hence we demand that all the components in the off-shell spectrum of the theory must be generated by superfields and perfectly organise into multiplets. We will call this the Superspace requirement.

Another key property these theories have, is that they describe free, massless irreps of one spin $j=Y+1 / 2$ and one $j=Y$. This means that among the components of the off-shell spectrum we must have (a.) the correct number and type of components we need for the correct off-shell description of exactly one spin $j=Y+1 / 2$ and exactly one spin $j=Y$ together with the appropriate gauge transformations, (b.) no other propagating d.o.f. We will call this requirement the Higher spin requirement.

The last requirement we demand is that of Economy. Although the previous three requirements define the type of theories we want to describe, they do not say anything about their complexity. So it is reasonable to ask for the most economical description of these systems. That means economy in terms of the number of superfields required, their rank as tensors and degrees of freedom.

In this paper we will show that by imposing the requirements of (i) Supersymmetry, (ii) Superspace, (iii) Higher spin and (iv) Economy as explained above we can determine the off-shell structure of the theory and the superspace description. The appeal of this approach is that it is very intuitive and, with great satisfaction, gives the answers obtained by following the technical constructions of [3].

The organization of the paper is the following. In sections 2, 3 and 4 we will derive the consequences of the above mentioned requirements. That will provide a classification of the various types of components and superfields we must consider as well as the type of gauge transformations they must have. Also, we derive a constraint on the number of components of each type, in the form of a Diophantine equation. In sections 5, 6 and 7 we apply these results to the vector multiplet $\left(Y=\frac{1}{2}\right)$, the matter-gravitino multiplet $(Y=1)$ and the supergravity multiplet $(Y=3 / 2)$. We find that this method will correctly generate all known formulations for these supermultiplets [one for the vector multiplet, two for the gravitino matter multiplet (de Wit-van Holten, Ogievetsky-Sokatchev) and four for the supergravity (old-minimal, new-minimal, new-new-minimal and non-minimal)]. In section 8 , we extend the construction to the arbitrary half-integer case $Y=s+1 / 2$ and correctly generate the two different ways to construct the supermultiplet in agreement with $[1,3]$. The arbitrary integer case $Y=s$ is being discussed in section 9 and we find that there is a unique answer in agreement with [3].

\section{On-shell data and the higher spin requirement}

Based on the group theoretic analysis of the irreducible representations of the superPoincaré group, we know that on-shell they must describe two successive spins, one integer and one half-integer. The off-shell description of these free, massless, higher spins is very well known [6-9]. Very briefly, in order to describe these higher spins irreps: 
$\alpha$ ) For integer spin $j=s$, we must have two real bosonic components $h_{\alpha(s) \dot{\alpha}(s)}$, $h_{\alpha(s-2) \dot{\alpha}(s-2)}$ with independently symmetrized dotted and undotted indices ${ }^{1}$ along with appropriate gauge transformations

$$
\begin{aligned}
\delta_{g} h_{\alpha(s) \dot{\alpha}(s)} & \sim \partial_{\left(\alpha _ { s } \left(\dot{\alpha}_{s} \zeta_{\alpha(s-1)) \dot{\alpha}(s-1))},\right.\right.} \\
\delta_{g} h_{\alpha(s-2) \dot{\alpha}(s-2)} & \sim \partial^{\beta \dot{\beta}} \zeta_{\beta \alpha(s-2) \dot{\beta} \dot{\alpha}(s-2)} .
\end{aligned}
$$

It is straightforward to count the off-shell degrees of freedom for spin $s$, the answer is $s^{2}+2 .^{2}$

$\beta$ ) For half-integer spin $j=s+1 / 2$, we must have three fermionic components $\psi_{\alpha(s+1) \dot{\alpha}(s)}$, $\psi_{\alpha(s) \dot{\alpha}(s-1)}$ and $\psi_{\alpha(s-1) \dot{\alpha}(s-2)}$ also with independently symmetrized indices along with gauge transformations

$$
\begin{aligned}
\delta_{g} \psi_{\alpha(s+1) \dot{\alpha}(s)} & \sim \partial_{\left(\alpha _ { s + 1 } \left(\dot{\alpha}_{s} \xi_{\alpha(s)) \dot{\alpha}(s-1))},\right.\right.} \\
\delta_{g} \psi_{\alpha(s) \dot{\alpha}(s-1)} & \sim \partial_{\left(\alpha_{s}\right.} \dot{\beta}_{\alpha(s-1)) \dot{\beta} \dot{\alpha}(s-1)}, \\
\delta_{g} \psi_{\alpha(s-1) \dot{\alpha}(s-2)} & \sim \partial^{\beta \dot{\beta}} \xi_{\beta \alpha(s-1) \dot{\beta} \dot{\alpha}(s-2)} .
\end{aligned}
$$

The counting of the off-shell degrees of freedom for spin $s+1 / 2$ is $4 s^{2}+4 s+4$.

Hence the off-shell spectrum of the higher spin supersymmetric theory must respect these structures and must include exactly one copy of these fields for each spin it describes onshell. Notice that the off-shell bosonic and fermionic degrees of freedom do not match. This is one way to realize that in supersymmetric theories we must introduce extra auxiliary fields in order to balance the bosonic and fermionic d.o.f, as requested by our Supersymmetry requirement.

\section{Demanding supersymmetry}

\subsection{Types of auxiliary fields}

The extra fields we have to add must not introduce any new propagating d.o.f, because the on-shell spectrum of the theory has already been taken care by the higher spin fields above. Therofore, the auxiliary fields must have no dynamics, hence they can be defined ${ }^{3}$ such that either they vanish on-shell or they are equivalent to zero up to gauge transformations. This mean that their equations of motion give a unique solution: ${ }^{4}$

$$
A=0 \text { or } A=0+\text { pure gauge. }
$$

The first one $[A=0]$ means that the equations of motion for these auxiliary fields are algebraic and therefore they must appear in the lagrangian in an algebraic manner. There

\footnotetext{
${ }^{1}$ The notation $A_{\alpha(k) \dot{\alpha}(l)}$ is a shorthand for $A_{\alpha_{1} \alpha_{2} \ldots \alpha_{k} \dot{\alpha}_{1} \dot{\alpha}_{2} \ldots \dot{\alpha}_{l}}$ which is a $(k, l)$-tensor with $k$ independently symmetrized undotted indices and $l$ independently symmetrized dotted indices.

${ }^{2}$ It may be useful to remind the reader that a $(k, l)$-tensor $A_{\alpha(k) \dot{\alpha}(l)}$ in $4 D$ has $2(k+1)(l+1)$ d.o.f. However if $k=l$ then we can talk about reality. A real $(k, k)$-tensor $A_{\alpha(k) \dot{\alpha}(k)}$ has $(k+1)^{2}$ d.o.f.

${ }^{3}$ After appropriate redefinitions to absorb

${ }^{4}$ For simplicity we suppressed all indices.
} 
are two ways this can happen, either they must appear in pairs $(A, B)[\mathcal{L}=\cdots+A B]$ with engineering dimensions $d_{A}+d_{B}=4,{ }^{5}$ or as singles $\left[\mathcal{L}=\cdots+A^{2}\right]$ with engineering dimensions $d_{A}=2$. Immediately we get that the fermionic auxiliary fields in this category must always appear in pairs, while the bosonic ones can appear as singles. Another important characteristic feature of these auxiliary fields is that all of them have to be gauge invariant $\left(\delta_{g} A=0\right)$ since the right hand side of the solution is gauge invariant.

The second type of solution $[A=0+$ pure gauge $]$ means the auxiliary fields have a non-trivial transformation $\delta_{g} A_{\ldots} \sim \partial_{\ldots} \ldots \lambda_{\ldots}$ with appropriate index contractions and symmetrizations to match the index structure of $A$. For this case, the equation of motion can be differential $(\mathcal{O} A=0$, where $\mathcal{O}$ is a differential operator) but it must have a unique solution which respects the gauge transformation of $A$, in other words the following must be true

$$
\mathcal{O} \cdot \partial \cdot \lambda=0 \quad \text { (identically) }
$$

thus putting $A$ in the same equivalence class as the zero solution. Assuming that $\mathcal{O}$ includes one derivative ${ }^{6}$ we obtain

$$
\partial \cdot \partial \cdot \lambda=0 \text { (identically) }
$$

where the dot $[\cdot]$ represent the various choices for contractions and symmetrization of the indices. We can go through the list of all options (all indices contracted, all indices symmetrizes, ...) but we quickly realize that in most of the cases the equation does not vanish identically. The way out is to realize that the identical nature of the equation must relay on the symmetries of the indices and since $\lambda$ must have symmetrized indices then the product $\partial \cdot \partial$ must generate an antisymmetrization. Such an identity exists

$$
\partial_{\alpha}{ }^{\dot{\alpha}} \partial_{\beta \dot{\alpha}} \sim C_{\alpha \beta} \square, \quad C_{\alpha \beta}=-C_{\beta \alpha} .
$$

Therefore, we conclude that $A$ must be of the form $A_{\alpha(k+1) \dot{\alpha}}$ and its equation of motion and gauge transformation are

$$
\partial_{\left(\alpha_{k+2}\right.}{ }^{\dot{\alpha}} A_{\alpha(k+1)) \dot{\alpha}}=0, \quad \delta_{g} A_{\alpha(k+1) \dot{\alpha}} \sim \partial_{\left(\alpha_{k+1} \dot{\alpha}\right.} \Lambda_{\alpha(k))}, \quad k \geq 0 .
$$

In order to generate this equation of motion from a lagrangian we must have the term

$$
\mathcal{L}=\cdots+B^{\alpha(k+2)} \partial_{\alpha_{k+2}} \dot{\alpha} A_{\alpha(k+1) \dot{\alpha}}+\text { c.c. }
$$

where $B_{\alpha(k+2)}$ is an extra auxiliary field with symmetrized indices. The equation of motion for $B$ is:

$$
\begin{aligned}
\partial^{\alpha_{k+2}} B_{\alpha(k+2)} & =0 \\
\text { or } & \\
\partial^{\alpha_{k+2}} B_{\alpha(k+2)} \pm \text { c.c. } & =0[\text { If we can impose a reality condition } A= \pm \bar{A}]
\end{aligned}
$$

\footnotetext{
${ }^{5}$ Keep in mind that the enginnering dimensions of bosonic fields is an integer number and for fermionic fields it is a half integer number. We have not proved that, but it will become obvious in the next section.

${ }^{6}$ Two or more derivatives will lead to dynamics which these fields must not have. For the same reason $A$ can not be a fermion because fermions have one derivative equations of motion.
} 
This equation must also be satisfied identically and give a solution that respects whatever gauge transformation $B_{\alpha(k+2)}$ has. We can repeat the arguments used in $A$ but we quickly realize they do not work in this case, because $B$ does not have dotted indices to symmetrize over, which was a consequence of the equation of $A$. So equation (3.7) can not be satisfied identically and the only hope for consistence is equation (3.8). For that to work $A$ has to be either real or imaginary, therefore the number of dotted and undotted indices of $A$ must match giving $k=0$. It is straight forward to show there is a unique solution of (3.8) and it gives the gauge transformation of $B_{\alpha \beta}$ to be

$$
\delta_{g} B_{\alpha \beta}=\partial_{(\alpha} \dot{\beta} L_{\beta) \dot{\beta}}, \quad L_{\beta \dot{\beta}}=\mp \bar{L}_{\beta \dot{\beta}}
$$

where $L_{\alpha \dot{\alpha}}$ is not uniquely defined because of the freedom

$$
L_{\beta \dot{\beta}} \sim L_{\beta \dot{\beta}}+\partial_{\beta \dot{\beta}} L, \quad L=\mp \bar{L} .
$$

As can seen from the above construction, there are no fermionic auxiliary fields that in this category.

To conclude, we find that for any massless, $4 D, \mathcal{N}=1$ theory, the supersymmetric auxiliary fields that appear in its off-shell spectrum organise in the following ways:

Fermions: There is only one kind of fermionic auxiliary feilds and have the properties

(i) they come in pairs $(\beta, \rho)\left[\mathcal{L}=\cdots+\beta \cdots \rho_{\ldots}+\right.$ c.c. $]$,

(ii) they are gauge invariant $\left[\delta_{g} \beta \ldots=0=\delta_{g} \rho_{\ldots} ..\right]$,

(iii) they have engineering dimensions $\frac{3}{2}$ and $\frac{5}{2}$.

Bosons: There are three types of auxiliary bosonic fields

Type (1): (i) they came in pairs of $\left(A_{\alpha \dot{\alpha}}, B_{\alpha \beta}\right)$ with $A_{\alpha \dot{\alpha}}= \pm \bar{A}_{\alpha \dot{\alpha}}\left[\mathcal{L}=\ldots+B^{\alpha \beta} \partial_{\alpha} \dot{\beta} A_{\beta \dot{\beta}}+\right.$ c.c. $]$,

(ii) they have non-trivial gauge transformations

$$
\begin{aligned}
{\left[\delta_{g} A_{\alpha \dot{\alpha}}\right.} & =\partial_{\alpha \dot{\alpha}} \lambda, \lambda= \pm \bar{\lambda} \\
\delta_{g} B_{\alpha \beta} & \left.=\partial_{(\alpha}{ }^{\dot{\beta}} L_{\beta) \dot{\beta}}, \delta_{g} L_{\beta \dot{\beta}}=\partial_{\beta \dot{\beta}} L, L_{\beta \dot{\beta}}=\mp \bar{L}_{\beta \dot{\beta}}, L=\mp \bar{L}\right]
\end{aligned}
$$

(iii) they have enginnering dimensions 2 and 1.

Type (2): (i) they are singles $\left[\mathcal{L}=\cdots+A \cdots A_{\ldots}+\right.$ c.c. $]$,

(ii) they are gauge invariant $\left[\delta_{g} A=0\right]$,

(iii) they have engineering dimensions 2.

Type (3): $(i)$ they come in pairs $(A, B)[\mathcal{L}=\cdots+A \cdots B \ldots+$ c.c. $]$,

(ii) they are gauge invariant $\left[\delta_{g} A_{\ldots}=0=\delta_{g} B_{\ldots}\right]$,

(iii) they have engineering dimensions 3 and 1 .

It is perhaps illustrative to expand on known examples of the Type (3) behavior already in the literature, though these are within the context of lower spin supermultiplets. The first 
work to demonstrate a pair of bosonic auxiliary fields with unequal engineering dimensions in a dynamical action with $\mathcal{N}=2$ supersymmetry was given in a work completed in 1983 [10]. Since then, a second such $\mathcal{N}=2$ supersymmetric system [11, 12] has been shown to exist. As one would expect, the work of [10] possesses an $\mathcal{N}=1$ truncation and this was done in the work of [13] which also gave a generalization where an arbitrary number of such pairs were shown to be possible for an $\mathcal{N}=1$ construction.

\subsection{Matching bosons with fermions}

Every bosonic auxiliary field will be a $(k, l)$-tensor with $k$-undotted and $l$-dotted indices $(k+$ $l=$ even) independently symmetrized. We have also shown that the bosonic auxiliary fields can be organized into classes $r$ based on their index structure, their gauge transformation and their reality or not. For each class of tensors we can count the off-shell degrees of freedom $\mathcal{D}_{r}$ they carry. Adding all these contributions, we can calculate the off-shell degrees of freedom that all auxiliary bosons carry

$$
\mathcal{A}_{B}=\sum_{r} \mathcal{D}_{r} \mathcal{N}_{r}^{B}
$$

The coefficient $\mathcal{N}_{r}$ is a multiplicity factor which counts the number of elements inside each class and the summation is over all possible classes. A similar computation can be done for the fermionic auxiliary fields and determine $\mathcal{A}_{F}$. Our Supersymmetry requirement to match the bosonic and fermionic off-shell d.o.f. can be expressed in the following way

$$
\left(\begin{array}{c}
\text { integer spin } \\
\text { off-shell d.o.f. }
\end{array}\right)+\mathcal{A}_{B}=\left(\begin{array}{c}
\text { half-integer spin } \\
\text { off-shell d.o.f. }
\end{array}\right)+\mathcal{A}_{F} \text {. }
$$

This is a Diophantine equation for the coefficients $\mathcal{N}_{r}^{B}$ and $\mathcal{N}_{r}^{F}$. Finding the values of these coefficients will determine completely the off-shell spectrum of the theory. Of course not all solutions of this Diophantine equation can have this interpretation and we will not blindly accept all solutions. However, we will show that the rest of our requirements will allow us to select only these solutions that correspond to manifestly supersymmetric theories. For example, the summation in (3.11) takes place over all possible types of auxiliary fields. Obviously there are many possibilities and the number of solutions that explore all of them becomes very big. Nevertheless, our Superspace requirement drastically reduces the allowed possibilities. When on top of that we add our demand for economy, then we are left with very little options. These surviving solutions will be the ones that can be realized as supersymmetric theories.

The logic of the arguments above seem "iron-clad," but it is useful to recall one example from the past demonstrating the possibility of a loop-hole in these arguments. In the work of [14], it was pointed out that sometimes a symmetry argument combined with arguments about degrees of freedom, can only be resolved by the essential use of non-linearity. In this case, the symmetry was Lorentz symmetry. However, it does act as a concrete example where relying solely on the linear realization leads one astray. With this caveat in mind, we will continue with our arguments under the assumption that we work in the domain of purely linear realizations. 


\section{Demanding existence of a superspace descriptions}

\subsection{Types of superfields}

The next requirement is that all fields must be generated from a set of superfields, meaning there exist an underlying superspace description that makes supersymmetry manifest even if we focus only on the component structure of the theory. Surely, this demand must put some constraint on the type of tensors that can emerge and therefore affect the balancing of the d.o.f. through (3.12).

Without loss of generality we can consider all superfields that participate in the superspace description of the theory to be unconstrained. If some of them are not, then we must be able to solve whatever constraint they satisfy and express them in terms of unconstrained ones. This is equivalent to demanding the existence of a set of so-called "prepotential superfields" that provide the most fundamental description of the theories in questions. For $4 \mathrm{D}, \mathcal{N}=1$ supersymmetric Yang-Mills theories, the prepotential is traditionally denoted by $V$, a real unconstrained pseudoscalar superfield. All geometrical structures such as connections and field strengths are determined by this superfield. For $4 \mathrm{D}, \mathcal{N}=1$ supergravity theories, the prepotential is often denoted by $U^{\gamma} \dot{\gamma}$, a real unconstrained axial vector superfield $[15,16]$ leading to a geometical formulation [17] of a curved supermanifold. Supersymmetrical generalizations of frame fields, spin-connection, and curvature tensor are all fundamentally defined by the $U^{\gamma \dot{\gamma}}$. It was later shown that the prepotential concept applied to supermultiplets with $Y=1$ [19]. As these supermultiplets are also gauged ones, it became obvious that this would be the case for all higher values of $Y$.

Let us consider an arbitrary, unconstrained superfield $\mathrm{A}_{\alpha(k) \dot{\alpha}(l)}$ with engineering dimensions $d_{\mathrm{A}}$. The kinetic energy term for this superfield will be quadratic in A and will have some number $N$ of spinorial covariant derivatives (D and $\overline{\mathrm{D}}$ )

$$
\int d^{8} z \mathrm{~A} \underbrace{\mathrm{D} \ldots \mathrm{D}}_{N} \mathrm{~A} \text { or } \int d^{8} z \mathrm{AD} \ldots \mathrm{D} \overline{\mathrm{A}} .
$$

Based on dimensional analysis we must have that

$$
N=4\left(1-d_{\mathrm{A}}\right)
$$

Also, it is easy to show that no matter what the index structure of A is, $N$ has to be even. Furthermore, $N$ has to be strictly positive, because otherwise A will have an algebraic equation of motion which means that it can be integrated out and therefore it is irrelevant. The result is $N \geq 2$, thus $d_{\mathrm{A}} \leq \frac{1}{2}$. The components $^{7}$ that such a superfield can generate are presented in table 1 .

Groups A and B are the groups of bosonic or the fermionic components depending if superfield $\mathrm{A}$ is bosonic $(k+l=$ even) or fermionic $(k+l=$ odd). All elements of

\footnotetext{
${ }^{7}$ The various components are labelled by the name of the superfield they come from and their position $(n, m)$ in its $\theta$ expansion. For example, $\Phi^{(0,0)}$ is the $\theta$ independent term of superfield $\Phi, \Phi_{\dot{\alpha}}^{(0,1)}$ is the $\bar{\theta}$ component and $\Phi_{\alpha \dot{\alpha}}^{(1,1)}$ is its $\theta \bar{\theta}$ component. Components with more than one index of the same type can be decomposed into symmetric (S) and anti-symmetric (A) pieces as $\Phi_{\beta \alpha}^{(S)}=\Phi_{(\beta \alpha)}, \Phi^{(A)}=C^{\beta \alpha} \Phi_{\beta \alpha}$.
} 


\begin{tabular}{|llll|}
\hline \multicolumn{2}{|c}{ Group A } & \multicolumn{2}{c|}{ Group B } \\
\hline$\left[d_{\mathrm{A}}\right]$ & $\mathrm{A}_{\alpha(k) \dot{\alpha}(l)}^{(0,0)}$ & {$\left[d_{\mathrm{A}}+\frac{1}{2}\right]$} & $\mathrm{A}_{\alpha(k+1) \dot{\alpha}(l)}^{(1,0)(S)}$ \\
{$\left[d_{\mathrm{A}}+1\right]$} & $\mathrm{A}_{\alpha(k) \dot{\alpha}(l)}^{(2,0)}$ & {$\left[d_{\mathrm{A}}+\frac{1}{2}\right]$} & $\mathrm{A}_{\alpha(k-1) \dot{\alpha}(l)}^{(1,0)(A)}$ \\
{$\left[d_{\mathrm{A}}+1\right]$} & $\mathrm{A}_{\alpha(k) \dot{\alpha}(l)}^{(0,2)}$ & {$\left[d_{\mathrm{A}}+\frac{1}{2}\right]$} & $\mathrm{A}_{\alpha(k) \dot{\alpha}(l+1)}^{(0,1)(S)}$ \\
{$\left[d_{\mathrm{A}}+1\right]$} & $\mathrm{A}_{\alpha(k+1) \dot{\alpha}(l+1)}^{(1,1)(S, S)}$ & {$\left[d_{\mathrm{A}}+\frac{1}{2}\right]$} & $\mathrm{A}_{\alpha(k) \dot{\alpha}(l-1)}^{(0,1)(A)}$ \\
{$\left[d_{\mathrm{A}}+1\right]$} & $\mathrm{A}_{\alpha(k+1) \dot{\alpha}(l-1)}^{(1,1)(S, A)}$ & {$\left[d_{\mathrm{A}}+\frac{3}{2}\right]$} & $\mathrm{A}_{\alpha(k+1) \dot{\alpha}(l)}^{(1,2)(S)}$ \\
{$\left[d_{\mathrm{A}}+1\right]$} & $\mathrm{A}_{\alpha(k-1) \dot{\alpha}(l+1)}^{(1,1)(A, S)}$ & {$\left[d_{\mathrm{A}}+\frac{3}{2}\right]$} & $\mathrm{A}_{\alpha(k-1) \dot{\alpha}(l)}^{(1,2)(A)}$ \\
{$\left[d_{\mathrm{A}}+1\right]$} & $\mathrm{A}_{\alpha(k-1) \dot{\alpha}(l-1)}^{(1,1)(A, A)}$ & {$\left[d_{\mathrm{A}}+\frac{3}{2}\right]$} & $\mathrm{A}_{\alpha(k) \dot{\alpha}(l+1)}^{(2,1)(S)}$ \\
{$\left[d_{\mathrm{A}}+2\right]$} & $\mathrm{A}_{\alpha(k) \dot{\alpha}(l)}^{(2,2)}$ & {$\left[d_{\mathrm{A}}+\frac{3}{2}\right]$} & $\mathrm{A}_{\alpha(k) \dot{\alpha}(l-1)}^{(2,1)(A)}$ \\
\hline
\end{tabular}

Table 1. List of fields generated by superfield A.

group A have the same grassman parity, equal to that of the superfield A and in contrast the elements of group $\mathrm{B}$ have the opposity grassman parity. If $\mathrm{A}$ is complex it carries $16 k l+16 k+16 l+16$ bosons and equal fermions, whereas if it is real (that also means $k=l$ ) it will carry $8 k^{2}+16 k+8$ bosons and equal fermions. An obvious observation is that the range of engineering dimensions for the components is from $d_{\mathrm{A}}$ to $d_{\mathrm{A}}+2$. Therefore in order for a superfield to provide propagating bosons and fermions we must have $d_{\mathrm{A}}+2 \geq \frac{3}{2} \Rightarrow d_{\mathrm{A}} \geq-\frac{1}{2}$. Therefore we conclude that the allowed superfields must have dimensions $d$ with

$$
-\frac{1}{2} \leq d \leq \frac{1}{2}
$$

So all bosonic superfields will have $d=0$ and the fermionic superfields will have $d=\frac{1}{2}$ or $d=-\frac{1}{2}$. The conclusion is:

$\alpha$ For $\mathcal{N}=1$ theories, bosonic auxiliary fields of type (3) are not permitted.

$\beta$ ) Fermionic auxiliary fields can only be generated by fermionic superfields with enginnering dimensions $d=\frac{1}{2}$.

\subsection{Gauge transformations}

It is a fact of physics that there is a discontinuity in the degrees of freedom of massless and massive theories and that insisting on describing the system in a local manner forces upon us the concept of redundancies (gauge symmetries). Hence, for massless theories there must be a gauge symmetry that can be lifted all the way to the superspace description. Here we discuss the various options for the superspace gauge symmetry of an arbitrary superfield $\mathrm{A}_{\alpha(k) \dot{\alpha}(l)}$.

First of all, the transformation of a superfield must not include algebraic terms bacause then we have the freedom to completely remove it, thus making it irrelevant. Therefore, 


\begin{tabular}{|c|c|c|c|c|}
\hline & $\mathrm{D}_{\left(\alpha_{k}\right.} L_{\alpha(k-1)) \dot{\alpha}(l)}$ & $\overline{\mathrm{D}}_{\left(\dot{\alpha}_{l}\right.} \Lambda_{\alpha(k) \dot{\alpha}(l-1))}$ & $\mathrm{D}^{\beta} J_{\beta \alpha(k) \dot{\alpha}(l)}$ & $\overline{\mathrm{D}}^{\dot{\beta}} I_{\alpha(k) \dot{\beta} \dot{\alpha}(l)}$ \\
\hline $\mathrm{A}_{\alpha(k) \dot{\alpha}(l)}^{(0,0)}$ & $\mathrm{D}_{\left(\alpha_{k}\right.} L_{\alpha(k-1)) \dot{\alpha}(l)} \mid$ & $\overline{\mathrm{D}}_{\left(\dot{\alpha}_{l}\right.} \Lambda_{\alpha(k) \dot{\alpha}(l-1))} \mid$ & $\mathrm{D}^{\beta} J_{\beta \alpha(k) \dot{\alpha}(l)} \mid$ & $\overline{\mathrm{D}}^{\dot{\beta}} I_{\alpha(k) \dot{\beta} \dot{\alpha}(l)} \mid$ \\
\hline $\mathrm{A}_{\alpha(k) \dot{\alpha}(l)}^{(2,0)}$ & 0 & $\mathrm{D}^{2} \overline{\mathrm{D}}_{\left(\dot{\alpha}_{l}\right.} \Lambda_{\alpha(k) \dot{\alpha}(l-1))} \mid$ & 0 & $\mathrm{D}^{2} \overline{\mathrm{D}}^{\dot{\beta}} I_{\alpha(k) \dot{\beta} \dot{\alpha}(l)} \mid$ \\
\hline $\mathrm{A}_{\alpha(k) \dot{\alpha}(l)}^{(0,2)}$ & $\overline{\mathrm{D}}^{2} \mathrm{D}_{\left(\alpha_{k}\right.} L_{\alpha(k-1)) \dot{\alpha}(l)} \mid$ & 0 & $\overline{\mathrm{D}}^{2} \mathrm{D}^{\beta} J_{\beta \alpha(k) \dot{\alpha}(l)} \mid$ & 0 \\
\hline $\mathrm{A}_{\alpha(k+1) \dot{\alpha}(l+1)}^{(1,1)(S, S)}$ & $i \partial_{\left(\alpha_{k+1}\left(\dot{\alpha}_{l+1}\right.\right.} \mathrm{D}_{\alpha_{k}} L_{\alpha(k-1)) \dot{\alpha}(l))} \mid$ & $i \partial_{\left(\alpha_{k+1}\left(\dot{\alpha}_{l+1}\right.\right.} \overline{\mathrm{D}}_{\dot{\alpha}_{l}} \Lambda_{\alpha(k)) \dot{\alpha}(l-1))} \mid$ & $\begin{array}{l}i \partial_{\left(\alpha_{k+1}\left(\dot{\alpha}_{l+1}\right.\right.} \mathrm{D}^{\beta} J_{\beta \alpha(k)) \dot{\alpha}(l))} \mid \\
\overline{\mathrm{D}}_{\left(\dot{\alpha}_{l+1}\right.} \mathrm{D}^{2} J_{\alpha(k+1) \dot{\alpha}(l))} \mid\end{array}$ & $\begin{array}{c}i \partial_{\left(\alpha_{k+1}\left(\dot{\alpha}_{l+1}\right.\right.} \overline{\mathrm{D}}^{\dot{\beta}} I_{\alpha(k)) \dot{\beta} \dot{\alpha}(l))} \mid \\
\mathrm{D}_{\left(\alpha_{k+1}\right.} \overline{\mathrm{D}}^{2} I_{\alpha(k)) \dot{\alpha}(l+1)} \mid\end{array}$ \\
\hline $\mathrm{A}_{\alpha(k+1) \dot{\alpha}(l-1)}^{(1,1)(S, A)}$ & $i \partial_{\left(\alpha_{k+1}\right.} \dot{\alpha}_{l} \mathrm{D}_{\alpha_{k}} L_{\alpha(k-1)) \dot{\alpha}(l)} \mid$ & 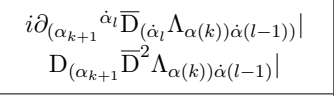 & $\begin{array}{c}i \partial_{\left(\alpha_{k+1}{ }_{l} \mathrm{D}^{\beta} J_{\beta \alpha(k)) \dot{\alpha}(l)} \mid\right.} \mid \\
\overline{\mathrm{D}}^{\dot{\alpha}_{l}} \mathrm{D}^{2} J_{\alpha(k+1) \dot{\alpha}(l)} \mid\end{array}$ & $i \partial_{\left(\alpha_{k+1}\right.} \dot{\gamma} \overline{\mathrm{D}}^{\dot{\beta}} I_{\alpha(k)) \dot{\gamma} \dot{\beta} \dot{\alpha}(l-1)} \mid$ \\
\hline $\mathrm{A}_{\alpha(k-1) \dot{\alpha}(l+1)}^{(1,1)(A, S)}$ & $\begin{array}{l}i \partial^{\alpha_{k}}{ }_{\left(\dot{\alpha}_{l+1}\right.} \mathrm{D}_{\left(\alpha_{k}\right.} L_{\alpha(k-1)) \dot{\alpha}(l))} \mid \\
\overline{\mathrm{D}}_{\left(\dot{\alpha}_{l+1} \mathrm{D}^{2} L_{\alpha(k-1) \dot{\alpha}(l))} \mid\right.}\end{array}$ & $i \partial_{\left(\dot{\alpha}_{l+1}\right.}^{\alpha_{k}} \overline{\mathrm{D}}_{\dot{\alpha}_{l}} \Lambda_{\alpha(k) \dot{\alpha}(l-1))} \mid$ & $i \partial_{\left(\dot{\alpha}_{l+1}\right.} \mathrm{D}^{\beta} J_{\gamma \beta \alpha(k-1) \dot{\alpha}(l))} \mid$ & $\begin{array}{c}i \partial_{\left(\dot{\alpha}_{l+1}\right.} \overline{\mathrm{D}}^{\dot{\beta}} I_{\gamma \alpha(k-1)) \dot{\beta} \dot{\alpha}(l))} \mid \\
\mathrm{D}^{\gamma} \overline{\mathrm{D}}^{2} I_{\gamma \alpha(k-1) \dot{\alpha}(l+1)} \mid\end{array}$ \\
\hline $\mathrm{A}_{\alpha(k-1) \dot{\alpha}(l-1)}^{(1,1)(A, A)}$ & $\begin{array}{c}i \partial^{\alpha_{k} \dot{\alpha}_{l}} \mathrm{D}_{\left(\alpha_{k}\right.} L_{\alpha(k-1)) \dot{\alpha}(l)} \mid \\
\overline{\mathrm{D}}^{\dot{\alpha}_{l}} \mathrm{D}^{2} L_{\alpha(k-1) \dot{\alpha}(l)} \mid\end{array}$ & $\begin{array}{c}i \partial^{\alpha_{k} \dot{\alpha}_{l}} \overline{\mathrm{D}}_{\left(\dot{\alpha}_{l}\right.} \Lambda_{\alpha(k) \dot{\alpha}(l-1))} \mid \\
\mathrm{D}^{\alpha_{k}} \overline{\mathrm{D}}^{2} \Lambda_{\alpha(k) \dot{\alpha}(l-1)} \mid\end{array}$ & $i \partial^{\gamma \dot{\gamma}} \mathrm{D}^{\beta} J_{\gamma \beta \alpha(k-1) \dot{\gamma} \dot{\alpha}(l-1)} \mid$ & $i \partial^{\gamma \dot{\gamma}} \overline{\mathrm{D}}^{\dot{\beta}} I_{\gamma \alpha(k-1) \dot{\gamma} \dot{\beta} \dot{\alpha}(l-1)} \mid$ \\
\hline $\mathrm{A}_{\alpha(k) \dot{\alpha}(l)}^{(2,2)}$ & $\begin{array}{c}i \partial^{\beta \dot{\beta}}\left[\mathrm{D}_{\beta}, \overline{\mathrm{D}}_{\dot{\beta}}\right] \mathrm{D}_{\left(\alpha_{k}\right.} L_{\alpha(k-1)) \dot{\alpha}(l)} \mid \\
\square \mathrm{D}_{\left(\alpha_{k}\right.} L_{\alpha(k-1) \dot{\alpha}(l)} \mid\end{array}$ & $\begin{array}{c}i \partial^{\beta \dot{\beta}}\left[\mathrm{D}_{\beta}, \overline{\mathrm{D}}_{\dot{\beta}}\right] \overline{\mathrm{D}}_{\left(\dot{\alpha}_{l}\right.} \Lambda_{\alpha(k) \dot{\alpha}(l-1))} \mid \\
\square \overline{\mathrm{D}}_{\left(\dot{\alpha}_{l}\right.} \Lambda_{\alpha(k) \dot{\alpha}(l-1))} \mid\end{array}$ & $\begin{array}{c}i \partial^{\gamma \dot{\gamma}}\left[\mathrm{D}_{\gamma}, \overline{\mathrm{D}}_{\dot{\gamma}}\right] \mathrm{D}^{\beta} J_{\beta \alpha(k) \dot{\alpha}(l)} \mid \\
\square \mathrm{D}^{\beta} J_{\beta \alpha(k) \dot{\alpha}(l)} \mid\end{array}$ & $\begin{array}{c}i \partial^{\gamma \dot{\gamma}}\left[\mathrm{D}_{\gamma}, \overline{\mathrm{D}}_{\dot{\gamma}}\right] \overline{\mathrm{D}}^{\dot{\beta}} I_{\alpha(k)) \dot{\beta} \dot{\alpha}(l))} \mid \\
\square \overline{\mathrm{D}}^{\dot{\beta}} I_{\alpha(k) \dot{\beta} \dot{\alpha}(l)} \mid\end{array}$ \\
\hline
\end{tabular}

Table 2. Group A.

there must be at least one spinorial covariant derivative and the most general gauge transformation of $\mathrm{A}_{\alpha(k) \dot{\alpha}(l)}$ can be parametrized in the following way: ${ }^{8}$

$$
\begin{aligned}
\delta \mathrm{A}_{\alpha(k) \dot{\alpha}(l)}= & \mathrm{D}_{\left(\alpha_{k}\right.} L_{\alpha(k-1)) \dot{\alpha}(l)}+\overline{\mathrm{D}}_{\left(\dot{\alpha}_{l}\right.} \Lambda_{\alpha(k) \dot{\alpha}(l-1))} \\
& +\mathrm{D}^{\beta} J_{\beta \alpha(k) \dot{\alpha}(l)}+\overline{\mathrm{D}}^{\dot{\beta}} I_{\alpha(k) \dot{\beta} \dot{\alpha}(l)}
\end{aligned}
$$

for some $L_{\alpha(k-1) \dot{\alpha}(l)}, \Lambda_{\alpha(k) \dot{\alpha}(l-1)}, J_{\alpha(k+1) \dot{\alpha}(l)}, I_{\alpha(k) \dot{\alpha}(l+1)}$. The first two terms consist what we will call a type $\mathcal{I}$ transformation and the last two terms is a type $\mathcal{I} \mathcal{I}$ transformation and any other type of gauge transformation can be generated by combining them and selecting appropriately $L, \Lambda, J, I$. Now we can deduce the gauge transformations for the various components of superfield A. These are presented in tables 2 and 3 for Group A and Group B components respectively.

These tables provide provide a list of the terms that appear in the transformation law of each of the components of A, for every possible type of gauge transformation. Notice that there are two types of terms. The terms that have spacetime derivatives and all the rest, which we will call algebraic because they include algebraic terms of components of the gauge parameters. ${ }^{9}$ The algebraic terms could be used to gauge remove the corresponding A component. In other words, the components that have only derivative terms in their transformation can not be removed. Such a term is the component $\mathrm{A}_{\alpha(k) \dot{\alpha}(l)}^{(2,2)}$. For any type of transformation, the coefficient of $\theta^{2} \bar{\theta}^{2}$ can never be gauged away and that is obvious because it has the maximum amount of $\theta$ s and $\bar{\theta}_{\mathrm{s}}$ hence it can not include algebraic terms in its transformation since the superfield transformation law is not algebraic. Therefore

\footnotetext{
${ }^{8}$ One must keep in mind that for the special case of $k=0$ or $l=0$ one must consider the presence of $\mathrm{D}^{2}$ or $\overline{\mathrm{D}}^{2}$ terms in the transformation law.

${ }^{9}$ One must be careful because the gauge parameters may have some further D-structure inside them that will convert the seemingly algebraic terms to derivative terms or make them vanish.
} 


\begin{tabular}{|c|c|c|c|c|}
\hline & $\mathrm{D}_{\left(\alpha_{k}\right.} L_{\alpha(k-1)) \dot{\alpha}(l)}$ & $\overline{\mathrm{D}}_{\left(\dot{\alpha}_{l}\right.} \Lambda_{\alpha(k) \dot{\alpha}(l-1))}$ & $\mathrm{D}^{\beta} J_{\beta \alpha(k) \dot{\alpha}(l)}$ & $\overline{\mathrm{D}}^{\dot{\beta}} I_{\alpha(k) \dot{\beta} \dot{\alpha}(l)}$ \\
\hline $\mathrm{A}_{\alpha(k+1) \dot{\alpha}(l)}^{(1,0)(S)}$ & 0 & $\mathrm{D}_{\left(\alpha_{k+1}\right.} \overline{\mathrm{D}}_{\left(\dot{\alpha}_{l}\right.} \Lambda_{\alpha(k)) \dot{\alpha}(l-1))} \mid$ & $\mathrm{D}^{2} J_{\alpha(k+1) \dot{\alpha}(l)} \mid$ & $\mathrm{D}_{\left(\alpha_{k+1}\right.} \overline{\mathrm{D}}^{\dot{\beta}} I_{\alpha(k)) \dot{\beta} \dot{\alpha}(l)} \mid$ \\
\hline $\mathrm{A}_{\alpha(k-1) \dot{\alpha}(l)}^{(1,0)(A)}$ & $\mathrm{D}^{2} L_{\alpha(k-1) \dot{\alpha}(l)} \mid$ & $\mathrm{D}^{\beta} \overline{\mathrm{D}}_{\left(\dot{\alpha}_{l}\right.} \Lambda_{\beta \alpha(k-1) \dot{\alpha}(l-1))} \mid$ & 0 & $\mathrm{D}^{\beta} \overline{\mathrm{D}}^{\dot{\beta}} I_{\beta \alpha(k-1) \dot{\beta} \dot{\alpha}(l)} \mid$ \\
\hline $\mathrm{A}_{\alpha(k) \dot{\alpha}(l+1)}^{(0,1)(S)}$ & $\overline{\mathrm{D}}_{\left(\dot{\alpha}_{l+1}\right.} \mathrm{D}_{\left(\alpha_{k}\right.} L_{\alpha(k-1)) \dot{\alpha}(l))} \mid$ & 0 & $\overline{\mathrm{D}}_{\left(\dot{\alpha}_{l+1}\right.} \mathrm{D}^{\beta} J_{\beta \alpha(k) \dot{\alpha}(l))} \mid$ & $\overline{\mathrm{D}}^{2} I_{\alpha(k) \dot{\alpha}(l+1)} \mid$ \\
\hline $\mathrm{A}_{\alpha(k) \dot{\alpha}(l-1)}^{(0,1)(A)}$ & $\overline{\mathrm{D}}^{\dot{\beta}} \mathrm{D}_{\left(\alpha_{k}\right.} L_{\alpha(k-1)) \dot{\beta} \dot{\alpha}(l-1)} \mid$ & $\overline{\mathrm{D}}^{2} \Lambda_{\alpha(k) \dot{\alpha}(l-1)} \mid$ & $\overline{\mathrm{D}}^{\dot{\beta}} \mathrm{D}^{\beta} J_{\beta \alpha(k) \dot{\beta} \dot{\alpha}(l-1)} \mid$ & 0 \\
\hline $\mathrm{A}_{\alpha(k+1) \dot{\alpha}(l)}^{(1,2)(S)}$ & $i \partial_{\left(\alpha_{k+1}\right.}{ }^{\dot{\beta}} \overline{\mathrm{D}}_{\dot{\beta}} \mathrm{D}_{\alpha_{k}} L_{\alpha(k-1)) \dot{\alpha}(l)}$ & $i \partial_{\left(\alpha_{k+1}\left(\dot{\alpha}_{l}\right.\right.} \overline{\mathrm{D}}^{2} \Lambda_{\alpha(k)) \dot{\alpha}(l-1))} \mid$ & $\begin{array}{c}i \partial_{\left(\alpha_{k+1}\right.}^{\dot{\beta}} \overline{\mathrm{D}}_{\dot{\beta}} \mathrm{D}^{\beta} J_{\beta \alpha(k)) \dot{\alpha}(l)} \mid \\
\overline{\mathrm{D}}^{2} \mathrm{D}^{2} J_{\alpha(k+1) \dot{\alpha}(l)} \mid\end{array}$ & $i \partial_{\left(\alpha_{k+1}\right.} \dot{\beta} \overline{\mathrm{D}}^{2} I_{\alpha(k)) \dot{\beta} \dot{\alpha}(l)} \mid$ \\
\hline $\mathrm{A}_{\alpha(k-1) \dot{\alpha}(l)}^{(1,2)(A)}$ & $\begin{array}{c}i \partial^{\alpha_{k}} \dot{\beta} \overline{\mathrm{D}}_{\dot{\beta}} \mathrm{D}_{\left(\alpha_{k}\right.} L_{\alpha(k-1)) \dot{\alpha}(l)} \\
\quad \overline{\mathrm{D}}^{2} \mathrm{D}^{2} L_{\alpha(k-1) \dot{\alpha}(l)} \mid\end{array}$ & $i \partial^{\beta}{ }_{\alpha_{l}} \overline{\mathrm{D}}^{2} \Lambda_{\beta \alpha(k-1) \dot{\alpha}(l-1))} \mid$ & $i \partial^{\beta \dot{\beta}} \overline{\mathrm{D}}_{\dot{\beta}} \mathrm{D}^{\gamma} J_{\gamma \beta \alpha(k-1) \dot{\alpha}(l)}$ & $i \partial^{\beta \dot{\beta}} \overline{\mathrm{D}}^{2} I_{\beta \alpha(k-1) \dot{\beta} \dot{\alpha}(l)} \mid$ \\
\hline $\mathrm{A}_{\alpha(k) \dot{\alpha}(l+1)}^{(2,1)(S)}$ & $i \partial_{\left(\alpha_{k}\left(\dot{\alpha}_{l+1}\right.\right.} \mathrm{D}^{2} L_{\alpha(k-1)) \dot{\alpha}(l))} \mid$ & $i \partial^{\beta}{ }_{\left(\dot{\alpha}_{l+1}\right.} \mathrm{D}_{\beta} \overline{\mathrm{D}}_{\dot{\alpha}_{l}} \Lambda_{\alpha(k) \dot{\alpha}(l-1))} \mid$ & $i \partial^{\beta}{ }_{\left(\dot{\alpha}_{l+1}\right.} \mathrm{D}^{2} J_{\beta \alpha(k) \dot{\alpha}(l))} \mid$ & $\begin{array}{c}i \partial^{\beta}{ }_{\left(\dot{\alpha}_{l+1}\right.} \mathrm{D}_{\beta} \overline{\mathrm{D}}^{\dot{\beta}} I_{\alpha(k) \dot{\beta} \dot{\alpha}(l))} \mid \\
\mathrm{D}^{2} \overline{\mathrm{D}}^{2} I_{\alpha(k) \dot{\alpha}(l+1)} \mid\end{array}$ \\
\hline $\mathrm{A}_{\alpha(k) \dot{\alpha}(l-1)}^{(2,1)(A)}$ & $i \partial_{\left(\alpha_{k}\right.}{ }^{\dot{\beta}} \mathrm{D}^{2} L_{\alpha(k-1)) \dot{\beta} \dot{\alpha}(l-1)} \mid$ & $\begin{array}{c}i \partial^{\beta \dot{\alpha}_{l}} \mathrm{D}_{\beta} \overline{\mathrm{D}}_{\left(\dot{\alpha}_{l}\right.} \Lambda_{\alpha(k) \dot{\alpha}(l-1)} \mid \\
\mathrm{D}^{2} \overline{\mathrm{D}}^{2} \Lambda_{\alpha(k) \dot{\alpha}(l-1)} \mid\end{array}$ & $i \partial^{\beta \dot{\beta}} \mathrm{D}^{2} J_{\beta \alpha(k) \dot{\beta} \dot{\alpha}(l-1)} \mid$ & $i \partial^{\beta \dot{\beta}} \mathrm{D}_{\beta} \overline{\mathrm{D}}^{\dot{\gamma}} I_{\beta \alpha(k-1) \dot{\beta} \dot{\gamma} \dot{\alpha}(l-1)} \mid$ \\
\hline
\end{tabular}

Table 3. Group B.

\begin{tabular}{|c|c|}
\hline If this is (partially) removed & Then these are (partially) removed as well \\
\hline $\mathrm{A}_{\alpha(k+1) \dot{\alpha}(l)}^{(1,2)(S)}$ & $\mathrm{A}_{\alpha(k+1) \dot{\alpha}(l)}^{(1,0)(S)}, \mathrm{A}_{\alpha(k) \dot{\alpha}(l+1)}^{(0,1)(S)}, \mathrm{A}_{\alpha(k) \dot{\alpha}(l-1)}^{(0,1)(A)}, \mathrm{A}_{\alpha(k) \dot{\alpha}(l)}^{(0,0)}, \mathrm{A}_{\alpha(k) \dot{\alpha}(l)}^{(0,2)}, \mathrm{A}_{\alpha(k+1) \dot{\alpha}(l+1)}^{(1,1)(S, S)}, \mathrm{A}_{\alpha(k+1) \dot{\alpha}(l-1)}^{(1,1)(S, A)}$ \\
\hline $\mathrm{A}_{\alpha(k-1) \dot{\alpha}(l)}^{(1,2)(A)}$ & $\mathrm{A}_{\alpha(k-1) \dot{\alpha}(l)}^{(1,0)(A)}, \mathrm{A}_{\alpha(k) \dot{\alpha}(l+1)}^{(0,1)(S)}, \mathrm{A}_{\alpha(k) \dot{\alpha}(l-1)}^{(0,1)(A)}, \mathrm{A}_{\alpha(k) \dot{\alpha}(l)}^{(0,0)}, \mathrm{A}_{\alpha(k) \dot{\alpha}(l)}^{(0,2)}, \mathrm{A}_{\alpha(k-1) \dot{\alpha}(l+1)}^{(1,1)(k, S)}, \mathrm{A}_{\alpha(k-1) \dot{\alpha}(l-1)}^{(1,1)(A, A)}$ \\
\hline $\mathrm{A}_{\alpha(k) \dot{\alpha}(l+1)}^{(2,1)(S)}$ & $\mathrm{A}_{\alpha(k+1) \dot{\alpha}(l)}^{(1,0)(S)}, \mathrm{A}_{\alpha(k-1) \dot{\alpha}(l)}^{(1,0)(A)}, \mathrm{A}_{\alpha(k) \dot{\alpha}(l)}^{(0,1)(S)}, \mathrm{A}_{\alpha(k) \dot{\alpha}(l)}^{(0,0)}, \mathrm{A}_{\alpha(k) \dot{\alpha}(l)}^{(2,0)}, \mathrm{A}_{\alpha(k+1) \dot{\alpha}(l+1)}^{(1,1)(S, S)}, \mathrm{A}_{\alpha(k-1) \dot{\alpha}(l+1)}^{(1,1)(A, S)}$ \\
\hline $\mathrm{A}_{\alpha(k) \dot{\alpha}(l-1)}^{(2,1)(f)}$ & $\mathrm{A}_{\alpha(k+1) \dot{\alpha}(l)}^{(1,0)(S)}, \mathrm{A}_{\alpha(k-1) \dot{\alpha}(l)}^{(1,0)(A)}, \mathrm{A}_{\alpha(k) \dot{\alpha}(l) 1)}^{(0,1)(A)}, \mathrm{A}_{\alpha(k) \dot{\alpha}(l)}^{(0,0)}, \mathrm{A}_{\alpha(k) \dot{\alpha}(l)}^{(2,0)}, \mathrm{A}_{\alpha(k+1) \dot{\alpha}(l-1)}^{(1,1)(S, A)}, \mathrm{A}_{\alpha(k-1) \dot{\alpha}(l-1)}^{(1,1)(A)}$ \\
\hline
\end{tabular}

Table 4. Conditional constraints.

for bosonic superfields $(d=0)$ this component will provide an auxiliary boson and for fermionic superfields with $d=\frac{1}{2}$ the $\mathrm{A}^{(2,2)}$ component gives the one member $(\beta)$ of the pair of auxiliary fermions.

The usefulness of these two tables is twofold. First of all, they introduce various associations among the components of a superfield. These associations, provide a set of conditional constraints that will help us reduce the number of possible solutions of the corresponding Diophantine equation. Specifically, we can deduce that if some component of a superfield can be gauged removed then there is a set of other components that can be removed as well and therefore a potential solution of the Diophantine equation that does not comply with these conditions can not have the interpretation of a manifest supersymmetric theory and will not be accepted. To generate the various sets of conditional constraints we focus at the components $\mathrm{A}_{\alpha(k+1) \dot{\alpha}(l)}^{(1,2)(S)}, \mathrm{A}_{\alpha(k-1) \dot{\alpha}(l)}^{(1,2)(A)}, \mathrm{A}_{\alpha(k) \dot{\alpha}(l+1)}^{(2,1)(S)}, \mathrm{A}_{\alpha(k) \dot{\alpha}(l-1)}^{(2,1)(A)}$. These components are special, because for each one of them there is a unique way to gauge remove them partially or fully and therefore they demand the presence of a specific term in the transformation law of the superfield. Hence we can safely conclude that there will be a set of other components that can be (partially) eliminated by the same term. The results are given in table 4 . 
The second use of tables 2 and 3 is to help us determine the gauge transformations we need to have given the set of components we find by solving the Diophantine equation.

\section{Demanding economy}

For theories without supersymmetry $(\mathcal{N}=0)$ given a tensor of some rank, it is natural to attempt to use it for the description of the highest possible spin it contains. From this point of view, the various on-shell constraints it must satisfy and the gauge transformation it has (for massless theories) it is just a way to remove the lower spin irreps it also contains. This is the preferred choice to describe any spin, although one could use an even higher rank tensor to describe lower spins, (e.g. [20] for an explicit discussion for such an approach). This approach involves a sense of economy, meaning that we introduce the minimal set of d.o.f required to describe a specific spin with all the symmetries manifest. Also it is one of the principles that lead to the off-shell spectrum of massless higher spins discussed in section 2. This economical description is now elevated to the $\mathcal{N}=1$ case and becomes one of our requirements. Therefore, we will search for the most economical solutions of the Diophantine equation in terms of the number of superfields we require to have, their rank and the degrees of freedom they carry off-shell. The consequences of having an economical description of a higher spin supersymmetric theory are:

i) the component that describes the highest spin must be unique and

ii) no other component tensor has higher rank.

In table 1 notice that the component $\mathrm{A}_{\alpha(k+1) \dot{\alpha}(l+1)}^{(1,1)(S, S)}$ is the highest rank component present and it is unique. These characteristics fit exactly to our demands, therefore for an economical description it makes sense to identify this component with the highest spin component of the on-shell spectrum. The consequence of this identification is to fix the values for the integers $k, l, d$ and the reality or not of the superfield that will carry the highest spin.

For example, if the highest spin is integer $s$ then we must have

$$
\begin{aligned}
\mathrm{A}_{\alpha(k+1) \dot{\alpha}(l+1)}^{(1,1)(S, S)} & \sim h_{\alpha(s) \dot{\alpha}(s)}, \\
k+1 & =s \\
l+1 & =s \\
d+1 & =1
\end{aligned}
$$

thus the superfield that will carry it ${ }^{10}$ must be a real bosonic superfield $H_{\alpha(s-1) \dot{\alpha}(s-1)}$ with $d_{H}=0$. Based on the results of the previous section we can immediatly say that one of the auxiliary bosons must be a real $(s-1, s-1)$-tensor which corresponds to the $H_{\alpha(s-1) \dot{\alpha}(s-1)}^{(2,2)}$. Also we can conclude that if any other superfield is required for the description of the theory must have less rank.

\footnotetext{
${ }^{10}$ The superfield that carries the highest spin, we will call it the main one.
} 
Similarly for the case of half-integer $s+\frac{1}{2}$ highest spin we must do the following identification

$$
\begin{aligned}
\mathrm{A}_{\alpha(k+1) \dot{\alpha}(l+1)}^{(1,1)(S, S)} & \sim \psi_{\alpha(s+1) \dot{\alpha}(s),}, \\
k+1 & =s+1, \\
l+1 & =s \\
d+1 & =\frac{3}{2} .
\end{aligned}
$$

The answer is that the main superfield must be a fermionic $\Psi_{\alpha(s) \dot{\alpha}(s-1)}$ with $d_{\Psi}=\frac{1}{2}$. Also, we conclude that there must be at least two auxiliary fermionic $(s, s-1)$-tensors which are gauge invariant, one of which corresponds to $\Psi_{\alpha(s) \dot{\alpha}(s-1)}^{(2,2)}$.

Furthermore, the highest spin component must have a specific gauge transformation. ${ }^{11}$ From table 2 we conclude that in order to match the expected higher spin transformation law, without having the risk to be able to gauge remove $\mathrm{A}_{\alpha(k+1) \dot{\alpha}(l+1)}^{(1,1)(S, S)}$, the main superfield that carries the highest spin component must have a transformation of type $\mathcal{I}$.

To gain a deeper appreciation of the restrictive nature of the use of the economical assumption, it is useful to recall the results in [2], where some of the results violate this assumption. To this point in time, this behaviour is only known to happen in a restricted class of the $(s, s+1 / 2)$ supermultiplets and is filtered out by the assumption of economic higher spin description.

\section{$6 \quad Y=1 / 2$ - vector supermultiplet}

In the previous three sections we presented the basic requirements we want to impose and derived their consequences. The next step is to apply them to various supermultiplets, starting with the vector supermultiplet, which has superspin $Y=\frac{1}{2}$. Now we go through the following steps:

1 - The dynamics involve a spin 1 and a spin $1 / 2$. The off-shell d.o.f. provided by the spins are

$$
\begin{aligned}
\operatorname{spin} 1: & \left.\left(s^{2}+2\right)\right|_{s=1}=3, \\
\text { spin 1/2: } & \left.\left(4 s^{2}+4 s+4\right)\right|_{s=0}=4 .
\end{aligned}
$$

2 - The highest spin is 1 , hence the main superfield in the superspace description must be a real scalar superfield $H$ with dimensions 0 , which also provides one real scalar auxiliary boson $\left(H^{(2,2)}\right)$. Because it is scalar it can not be of type (1) hence it must be of type (2) and carries 1 off-shell d.o.f. $\Rightarrow \mathcal{A}_{B}=1$ and $\mathcal{A}_{F}=0$.

3 - We check for the matching of bosons with fermions

$$
3+1=4+0
$$

\footnotetext{
${ }^{11}$ See section 2 .
} 
and since they do match, we do not have to add any more auxiliary components. So the theory will be described only by the main superfield $H$.

4 - The gauge transformation of $H$ is determined by the fact that the component $H^{(0,0)}$, $H^{(2,0)}, H_{\alpha}^{(1,0)}$ must be gauge removed, component $H^{(2,2)}$ must be gauge invariant and the reality condition of $H$. The unique answer is

$$
\delta_{g} H=\mathrm{D}^{2} \bar{L}+\overline{\mathrm{D}}^{2} L .
$$

\section{$7 \quad Y=1$ - matter-gravitino supermultiplets}

Next is the matter-gravitino supermultiplets with $Y=1$. In this case we have:

1 - The dynamic part of the theory is that of free spins $3 / 2$ and 1 , which provide the following number of off-shell d.o.f.

$$
\begin{array}{rr}
\text { spin } 1: & \left.\left(s^{2}+2\right)\right|_{s=1}=3, \\
\text { spin 3/2: } & \left.\left(4 s^{2}+4 s+4\right)\right|_{s=1}=12 .
\end{array}
$$

2 - The highest spin is $3 / 2$, therefore the main superfield must be a $(1,0)$-tensor $\Psi_{\alpha}$ of engineering dimensions $1 / 2$ and it will provide a fermionic auxiliary component $\Psi_{\alpha}^{(2,2)}$ with dimensions $\frac{5}{2}$ and since we know that fermionic auxiliary fields come in pairs, there must

\begin{tabular}{|c|c|c|c|c|c|}
\hline \multirow[t]{8}{*}{$1 \times \Psi_{\alpha},\left[\Psi_{\alpha}\right]=\frac{1}{2}$} & Fermions: & $\begin{array}{l}\text { Tensor } \\
(1,0) \\
(1,0)\end{array}$ & $\begin{array}{c}\text { Dimensions } \\
{\left[\frac{5}{2}\right]} \\
{\left[\frac{3}{2}\right]}\end{array}$ & $\begin{array}{c}\text { off-shell d.o.f. } \\
\qquad \begin{array}{c}4 \\
4\end{array}\end{array}$ & $\begin{array}{c}\text { multiplicity } \\
\quad 1 \\
\leq 3\end{array}$ \\
\hline & Bosons: & $(2,0)$ & {$[2]$} & 6 [type (2)] & $\leq 1$ \\
\hline & & & {$[2]$} & 3 [type (1)] & $\leq 1$ \\
\hline & & & {$[1]$} & 3 [type (1)] & $\leq 1$ \\
\hline & & $(0,0)$ & {$[2]$} & 1 [type (2)] & $\leq 2$ \\
\hline & & $(1,1)$ & {$[2]$} & 4 [type (2)] & $\leq 2$ \\
\hline & & & {$[2]$} & 3 [type (1)] & $\leq 2$ \\
\hline & & & {$[1]$} & 3 [type (1)] & $\leq 2$ \\
\hline
\end{tabular}
be another fermionic auxiliary component with the same index structure and dimensions $\frac{3}{2}$. In detail, superfield $\Psi_{\alpha}$ has the following list of potential auxiliary components:

where the last column gives the number of times a specific type of a component appears. If the theory requires extra fields, respecting the uniqueness of higher spin, then these extra superfields must be lower rank tensors. In this case there is no alternative but for them to be $\mathcal{N}_{V}$ copies of a real scalar. The potential auxiliary components they can provide are:

\begin{tabular}{|cccccc|}
\hline $\mathcal{N}_{V} \times V,[V]=0:$ & & Tensor & Dimensions & off-shell d.o.f. & multiplicity \\
& Bosons: & $(0,0)$ & {$[2]$} & 1 [type $(2)]$ & $\mathcal{N}_{V}$ \\
& & $(1,1)$ & {$[1]$} & 3 [type $(1)]$ & $\leq \mathcal{N}_{V}$ \\
\hline
\end{tabular}


3 - Matching bosons with fermions.

For fermions we can immediately write that $\mathcal{A}_{F}=8$, since there is a non-removable $(1,0)$ component coming from $\Psi_{\alpha}$ and we know that auxiliary fermions must appear in pairs. Therefore, the total number of off-shell d.o.f for this supermultiplet will be $12+8=20$ and the rest of potential auxiliary fermionic components must be removed by an appropriate gauge symmetry. For the bosons we have to consider the two different types we can have [type (1) and type (2)]. The potential type (2) auxiliary fields are:

$$
\begin{array}{ll}
\mathcal{N}_{(2,0)}=\mathcal{N}_{(2,0)}^{\Psi}, & 0 \leq \mathcal{N}_{(2,0)}^{\Psi} \leq 1, \\
\mathcal{N}_{(1,1)}=\mathcal{N}_{(1,1)}^{\Psi}, & 0 \leq \mathcal{N}_{(1,1)}^{\Psi} \leq 2, \\
\mathcal{N}_{(0,0)}=\mathcal{N}_{(0,0)}^{\Psi}+\mathcal{N}_{V}, & 0 \leq \mathcal{N}_{(0,0)}^{\Psi} \leq 2,
\end{array}
$$

where $\mathcal{N}_{(2,0)}$ is the number of $(2,0)$-tensors, $\mathcal{N}_{(1,1)}$ is the number of real $(1,1)$-tensors, $\mathcal{N}_{(0,0)}$ is the number of real $(0,0)$-tensors and the above expressions give their decomposition to contributions coming from the various superfields. For type (1), we have fields with two different dimensions so we get:

$$
\begin{aligned}
& \mathcal{K}_{[2]}=\mathcal{K}_{(2,0)}^{\Psi}+\mathcal{K}_{(1,1)}^{\Psi}, \\
& \mathcal{K}_{[1]}=k_{(2,0)}^{\Psi}+k_{(1,1)}^{\Psi}+k_{(1,1)}^{V},
\end{aligned}
$$

where $\mathcal{K}_{[2]}$ is the contribution of superfields to type (1) fields with engineering dimension two and similarly $\mathcal{K}_{[1]}$ for dimension one. Because they come in pairs it must be true that:

$$
\begin{aligned}
& \mathcal{K}_{(2,0)}^{\Psi}=k_{(1,1)}^{\Psi}+k_{(1,1)}^{V}, \\
& \mathcal{K}_{(1,1)}^{\Psi}=k_{(2,0)}^{\Psi}
\end{aligned}
$$

and therefore

$$
\mathcal{K} \equiv \mathcal{K}_{[2]}=\mathcal{K}_{[1]} .
$$

Similarly with the type (2) contributions, all of the type (1) contributions have appropriate upper bounds which state that we can not have more than what it is provided by the superfields. Among these constraints, two are special. As we can see from the tables above there can be only one $(2,0)$-tensor with dimensions 2 , therefore we must have

$$
\mathcal{N}_{(2,0)}^{\Psi}+\mathcal{K}_{(2,0)}^{\Psi} \leq 1 .
$$

A similar argument applies to $(1,1)$-tensors with dimension 2 , thus we get

$$
\mathcal{N}_{(1,1)}^{\Psi}+\mathcal{K}_{(1,1)}^{\Psi} \leq 2 .
$$

However, there is an important observation we should do. Out of all type (1), (1,1)-tensors with dimensions 1 we can generate, one of them will play the role of the propagating spin 1. Because the spin 1 contribution to the total bosonic d.o.f has been taken into account by (7.1) we should not count it again. This is a very special situation that arises only when we describe spin 1 propagating d.o.f in the presence of type (1) auxiliary fields. 
That is because the type (1) auxiliary components have the same structure and gauge transformation law with the spin 1 component and for that reason we have to be cautious in order to guarantee the presence of an appropriate spin one components. Obviously, this is not going to be an issue for higher spins. Putting everything together we get that

$$
\mathcal{A}_{B}=6 \mathcal{N}_{(2,0)}+4 \mathcal{N}_{(1,1)}+\mathcal{N}_{(0,0)}+6 \mathcal{K}
$$

and therefore the condition of matching the bosonic d.o.f with the fermionic ones takes the form

$$
3+\mathcal{A}_{B}=12+\mathcal{A}_{F} .
$$

This condition can be written in the following way

$$
6\left[\mathcal{N}_{(2,0)}^{\Psi}+\mathcal{K}_{(2,0)}^{\Psi}+\mathcal{K}_{(1,1)}^{\Psi}\right]+4 \mathcal{N}_{(1,1)}^{\Psi}+\mathcal{N}_{(0,0)}^{\Psi}+\mathcal{N}_{V}=17
$$

This is the Diophantine equation we have to solve together with all the various inequalities that constraint the value of the various coefficients.

\section{Solutions.}

(i) First of all, we have to check whether there is a solution without the need for extra superfields, meaning $\mathcal{N}_{V}=0$. In this case we have $k_{(1,1)}^{V}=0$ which also means that the physical spin 1 d.o.f will come out of the real or imaginary part of $\Psi_{\alpha \dot{\alpha}}^{(0,1)(S)}$. According to table 3, this can happen if $\Psi_{\alpha}$ has a transformation that includes the term $\delta \Psi_{\alpha}=\mathrm{D}_{\alpha} K$, where $K$ is either real or imaginary. The consequence is that the other part of $\Psi_{\alpha \dot{\alpha}}^{(0,1)(S)}$ can be eliminated and therefore we get the conditions

$$
\mathcal{K}_{(2,0)}^{\Psi}=k_{(1,1)}^{\Psi}=0 .
$$

Wit all the above in mind and due to the inequalities (7.11), (7.12), (7.5) we can prove that there exist a unique solution

$$
\mathcal{N}_{(2,0)}^{\Psi}=1, \mathcal{N}_{(1,1)}^{\Psi}=1, \mathcal{N}_{(0,0)}^{\Psi}=1, \mathcal{K}_{(1,1)}^{\Psi}=1, k_{(2,0)}^{\Psi}=1
$$

This solution correspondes to the Ogievetsky-Sokatchev description of the $\left(\frac{3}{2}, 1\right)$ multiplet [22] and the off-shell spectrum of the theory is:

1. one $(2,0)$-tensor of type $(2)$ :

$$
t_{\alpha \beta}, \delta_{g} t_{\alpha \beta}=0,\left[t_{\alpha \beta}\right]=2,
$$

2. one real $(1,1)$-tensor of type $(2)$ :

$$
A_{\alpha \dot{\alpha}}, A_{\alpha \dot{\alpha}}=\bar{A}_{\alpha \dot{\alpha}}, \delta_{g} A_{\alpha \dot{\alpha}}=0,\left[A_{\alpha \dot{\alpha}}\right]=2,
$$

3. one real $(0,0)$-tensor of type $(2)$ :

$$
P, P=\bar{P}, \delta_{g} P=0,[P]=2,
$$


4. one real $(1,1)$-tensor of type (1):

$$
V_{\alpha \dot{\alpha}}, V_{\alpha \dot{\alpha}}=\bar{V}_{\alpha \dot{\alpha}}, \delta_{g} V_{\alpha \dot{\alpha}}=\partial_{\alpha \dot{\alpha}} \lambda, \lambda=\bar{\lambda},\left[V_{\alpha \dot{\alpha}}\right]=2,
$$

5. one (2,0)-tensor of type (1):

$$
\omega_{\alpha \beta}, \delta_{g} \omega_{\alpha \beta}=i \partial_{(\beta}{ }^{\dot{\alpha}} \ell_{\alpha) \dot{\alpha}}, \ell_{\alpha \dot{\alpha}}=\bar{\ell}_{\alpha \dot{\alpha}}, \ell_{\alpha \dot{\alpha}} \sim \ell_{\alpha \dot{\alpha}}+\partial_{\alpha \dot{\alpha}} \ell, \ell=\bar{\ell},\left[\omega_{\alpha \beta}\right]=1 .
$$

(ii) Let us investigate the existence of solutions with $\mathcal{N}_{V} \neq 0$. Following the requirement of economy, we will assume that $\mathcal{N}_{V}$ takes the least possible value and if this is not enough to solve (7.15) then we will increase its value by one and check again. So for $\mathcal{N}_{V}=1$ we get:

$$
\begin{aligned}
6\left[\mathcal{N}_{(2,0)}^{\Psi}+\mathcal{K}_{(2,0)}^{\Psi}+\mathcal{K}_{(1,1)}^{\Psi}\right]+4 \mathcal{N}_{(1,1)}^{\Psi}+\mathcal{N}_{(0,0)}^{\Psi} & =16 \\
\mathcal{N}_{(2,0)}^{\Psi}+\mathcal{K}_{(2,0)}^{\Psi} & \leq 1 \\
\mathcal{N}_{(1,1)}^{\Psi}+\mathcal{K}_{(1,1)}^{\Psi} & \leq 2 \\
\mathcal{N}_{(0,0)}^{\Psi} & \leq 2 .
\end{aligned}
$$

This system has two solutions:

$$
\begin{aligned}
& (\alpha) \quad \mathcal{N}_{(2,0)}^{\Psi}+\mathcal{K}_{(2,0)}^{\Psi}=1, \mathcal{K}_{(1,1)}^{\Psi}=1, \mathcal{N}_{(1,1)}^{\Psi}=1, \mathcal{N}_{(0,0)}^{\Psi}=0 \\
& (\beta) \quad \mathcal{N}_{(2,0)}^{\Psi}+\mathcal{K}_{(2,0)}^{\Psi}=1, \mathcal{K}_{(1,1)}^{\Psi}=0, \mathcal{N}_{(1,1)}^{\Psi}=2, \mathcal{N}_{(0,0)}^{\Psi}=2 .
\end{aligned}
$$

However solution $(\alpha)$, due to $\mathcal{N}_{(0,0)}^{\Psi}=0$, demands part of the transformation of $\Psi_{\alpha}$ to include a term $\mathrm{D}_{\alpha} L$ for an unconstrained $L$. The result is that component $\Psi_{\alpha \dot{\alpha}}^{(0,1)(S)}$ can be completely removed $\left(k_{(1,1)}^{\Psi}=0\right)$ and therefore the spin 1 description must come from $V_{\alpha \dot{\alpha}}^{(1,1)}\left(k_{(1,1)}^{V}=0\right)$. Hence we must have $\mathcal{K}_{(2,0)}^{\Psi}=0$. The result is that the off-shell spectrum for solution $(\alpha)$ is identical to that of case $(i)$ with $\mathcal{N}_{V}=0$. So we get that same theory using one extra superfield and for that reason this solution is rejected. The lesson here is that it is important to develop a method that will help us filter out equivalent theories and give only the different descriptions. More on that in the following section.

Solution $(\beta)$ is a genuine different off-shell description of the same multiplet with a different spectrum. Using similar argument as before, we can show that $\mathcal{K}_{(2,0)}^{\Psi}=0$ and therefore the list of off-shell auxiliary components required are:

1. one (2,0)-tensor of type (2):

$$
t_{\alpha \beta}, \delta_{g} t_{\alpha \beta}=0,\left[t_{\alpha \beta}\right]=2,
$$

2. two real $(1,1)$-tensors of type (2):

$$
\begin{aligned}
& A_{\alpha \dot{\alpha}}, A_{\alpha \dot{\alpha}}=\bar{A}_{\alpha \dot{\alpha}}, \delta_{g} A_{\alpha \dot{\alpha}}=0,\left[A_{\alpha \dot{\alpha}}\right]=2, \\
& U_{\alpha \dot{\alpha}}, U_{\alpha \dot{\alpha}}=\bar{U}_{\alpha \dot{\alpha}}, \delta_{g} U_{\alpha \dot{\alpha}}=0,\left[U_{\alpha \dot{\alpha}}\right]=2,
\end{aligned}
$$


3. three real $(0,0)$-tensor of type $(2)$ :

$$
\begin{aligned}
& P, P=\bar{P}, \delta_{g} P=0,[P]=2, \\
& S, S=\bar{S}, \delta_{g} S=0,[S]=2, \\
& L, L=\bar{L}, \delta_{g} L=0,[L]=2 .
\end{aligned}
$$

This solution corresponds to the de Wit-van Holten description of the $\left(\frac{3}{2}, 1\right)$ multiplet $[18,19]$ and in contrast to the $\mathcal{N}_{V}=0$ case, there are no type (1) bosonic components.

4 - The gauge transformation of the superfields for the two descriptions $(i)$ and $(i i)(\beta)$ can be found in straight forward manner by looking at tables 2 and 3 and demanding the non participating components to be gauge removed and the components that describe the spin d.o.f to have the proper transformation laws. For case $(i)$ we showed that we need to have a term $\mathrm{D}_{\alpha} K$, where $K$ is either real or imaginary in order to make sure of the presence of spin one. Also, because we must have $\mathcal{N}_{(0,0)}^{\Psi}=1$, either the real or the imaginary part of component $\Psi^{(1,0)(A)}$ must be gauged away without removing the other one. It is obvious from table 3 , that this is possible if part of the transformation law of $\Psi_{\alpha}$ is $\delta \Psi_{\alpha}=\overline{\mathrm{D}}^{2} \mathrm{D}_{\alpha} \Lambda$ with $\Lambda$ either real or purely imaginary. Hence putting these two together we get

$$
\delta \Psi_{\alpha}=\mathrm{D}_{\alpha} K_{1}+i \overline{\mathrm{D}}^{2} \mathrm{D}_{\alpha} K_{2}, \quad K_{i}=\bar{K}_{i} .
$$

For solution $(i i)(\beta)$ removing all the uneccessary components give

$$
\begin{aligned}
\delta \Psi_{\alpha} & =\mathrm{D}^{2} K_{\alpha}+\overline{\mathrm{D}}^{2} K_{\alpha}, \\
\delta V & =\mathrm{D}^{\alpha} K_{\alpha}+\overline{\mathrm{D}}^{\dot{\alpha}} \bar{K}_{\dot{\alpha}} .
\end{aligned}
$$

\section{$8 \quad Y=3 / 2-$ supergravity supermultiplets}

We repeat the steps for the supergravity supermultiplet:

1 - The dynamics are that of spin 2 and 3/2, therefore the corresponding off-shell d.o.f. are

$$
\begin{array}{rr}
\text { spin 2: }\left.\quad\left(s^{2}+2\right)\right|_{s=2}=6, \\
\text { spin 3/2: } & \left.\left(4 s^{2}+4 s+4\right)\right|_{s=1}=12 .
\end{array}
$$

2 - The highest spin is 2 , therefore the main superfield must be a real $(1,1)$-tensor $H_{\alpha \dot{\alpha}}$ of zero dimensions which provides a real vector auxiliary boson which could be of type (1) or of type (2). We also know that $H_{\alpha \dot{\alpha}}$ must have a gauge transformation of type $\mathcal{I}$. Furthermore, all of its components with engineering dimensions less or equal than $\frac{1}{2}$ must be able to be gauged away. That means the components $H^{(1,0)(S)}, H^{(1,0)(A)}, H^{(0,1)(S)}$, $H^{(0,1)(A)}$ must have algebraic terms in their transformations, hence according to table 2 we note the gauge transformation of $H_{\alpha \dot{\alpha}}$ must be

$$
\delta_{g} H_{\alpha \dot{\alpha}}=\mathrm{D}_{\alpha} \bar{L}_{\dot{\alpha}}-\overline{\mathrm{D}}_{\dot{\alpha}} L_{\alpha}
$$

with $L_{\alpha}$ unconstrained. 
3 - Checking the matching of bosonic and fermionic d.o.f.

If the only auxiliary field is $H_{\alpha \dot{\alpha}}^{(2,2)}$, then it must be of type (2), hence $\mathcal{A}_{B}=4, \mathcal{A}_{F}=0$. However, because

$$
6+4 \neq 12+0
$$

this can not be the case and we have to introduce extra fields. This means that in the superspace description there must be more superfields besides the main one. Respecting the uniqueness of the highest spin, these extra superfields must be lower rank tensors, thus the only options we have are $\mathcal{N}_{\chi}$ copies of a fermionic superfield $\chi_{\alpha}$ with dimension $\frac{1}{2}, \mathcal{N}_{\psi}$ copies of a fermionic superfield $\psi_{\alpha}$ with dimension $-\frac{1}{2}$ and $\mathcal{N}_{V}$ copies of a real bosonic superfield $V$ with dimension 0 . Let us analyze the various auxiliary fields they can contribute:

\begin{tabular}{|c|c|c|c|c|c|}
\hline $1 \times H_{\alpha \dot{\alpha}},\left[H_{\alpha \dot{\alpha}}\right]=0$ & Bosons: & $\begin{array}{l}\text { Tensor } \\
(1,1)\end{array}$ & $\begin{array}{c}\text { Dimensions } \\
{[2]} \\
{[2]}\end{array}$ & $\begin{array}{c}\text { off-shell d.o.f. } \\
4 \text { [type }(2)] \\
3 \text { [type }(1)]\end{array}$ & $\begin{array}{c}\text { multiplicity } \\
\quad \leq 1 \\
\leq 1\end{array}$ \\
\hline \multirow[t]{8}{*}{$\mathcal{N}_{\chi} \times \chi_{\alpha},\left[\chi_{\alpha}\right]=\frac{1}{2}$} & Fermions: & $(1,0)$ & {$\left[\frac{5}{2}\right]$} & 4 & $\mathcal{N}_{\chi}$ \\
\hline & Bosons: & $(2,0)$ & {$[2]$} & 6 [type (2)] & $\leq \mathcal{N}_{\chi}$ \\
\hline & & & {$[2]$} & 3 [type (1)] & $\leq \mathcal{N}_{\chi}$ \\
\hline & & & {$[1]$} & 3 [type (1)] & $\leq \mathcal{N}_{\chi}$ \\
\hline & & $(0,0)$ & {$[2]$} & 1 [type (2)] & $\leq 2 \mathcal{N}_{\chi}$ \\
\hline & & $(1,1)$ & {$[2]$} & 4 [type (2)] & $\leq 2 \mathcal{N}_{\chi}$ \\
\hline & & & {$[2]$} & 3 [type (1)] & $\leq 2 \mathcal{N}_{\chi}$ \\
\hline & & & {$[1]$} & 3 [type (1)] & $\leq 2 \mathcal{N}_{\chi}$ \\
\hline \multirow[t]{2}{*}{$\mathcal{N}_{\psi} \times \psi_{\alpha},\left[\psi_{\alpha}\right]=-\frac{1}{2}$} & Bosons: & $(2,0)$ & {$[1]$} & 3 [type (1)] & $\leq \mathcal{N}_{\psi}$ \\
\hline & & $(1,1)$ & {$[1]$} & 3 [type (1)] & $\leq \mathcal{N}_{\psi}$ \\
\hline \multirow[t]{2}{*}{$\mathcal{N}_{V} \times V,[V]=0$} & Bosons: & $(0,0)$ & {$[2]$} & 1 [type (2)] & $\mathcal{N}_{V}$ \\
\hline & & $(1,1)$ & {$[1]$} & 3 [type (1)] & $\leq \mathcal{N}_{V}$ \\
\hline
\end{tabular}

For fermions, there is a non-removable $(1,0)$ component coming from every copy of $\chi_{\alpha}$ with the correct dimensions and because fermionic auxiliary fields come in pairs, we can immediately write that

$$
\mathcal{A}_{F}=8 \mathcal{N}_{\chi}
$$

For bosons, we have to consider both types (1) and (2). Potential type (2) contributions are

$$
\begin{aligned}
& \mathcal{N}_{(2,0)}=\mathcal{N}_{(2,0)}^{\chi}, \quad 0 \leq \mathcal{N}_{(2,0)}^{\chi} \leq \mathcal{N}_{\chi}, \\
& \mathcal{N}_{(1,1)}=\mathcal{N}_{(1,1)}^{\chi}+\mathcal{N}_{(1,1)}^{H}, \quad 0 \leq \mathcal{N}_{(1,1)}^{\chi} \leq 2 \mathcal{N}_{\chi}, \quad 0 \leq \mathcal{N}_{(1,1)}^{H} \leq 1, \\
& \mathcal{N}_{(0,0)}=\mathcal{N}_{(0,0)}^{\chi}+\mathcal{N}_{(0,0)}^{V}, \quad 0 \leq \mathcal{N}_{(0,0)}^{\chi} \leq 2 \mathcal{N}_{\chi}, \quad \mathcal{N}_{(0,0)}^{V}=\mathcal{N}_{V} .
\end{aligned}
$$

For the type (1) auxiliary bosons we get:

$$
\begin{aligned}
& \mathcal{K}_{[2]}=\mathcal{K}_{(1,1)}^{H}+\mathcal{K}_{(1,1)}^{\chi}+\mathcal{K}_{(2,0)}^{\chi}, \\
& \mathcal{K}_{[1]}=k_{(1,1)}^{\chi}+k_{(2,0)}^{\chi}+k_{(1,1)}^{\psi}+k_{(2,0)}^{\psi}+k_{(1,1)}^{V} .
\end{aligned}
$$


Because they come in pairs it must be true that

$$
\begin{aligned}
\mathcal{K}_{(1,1)}^{H}+\mathcal{K}_{(1,1)}^{\chi} & =k_{(2,0)}^{\chi}+k_{(2,0)}^{\psi}, \\
\mathcal{K}_{(2,0)}^{\chi} & =k_{(1,1)}^{\chi}+k_{(1,1)}^{\psi}+k_{(1,1)}^{V}, \\
\mathcal{K} \equiv \mathcal{K}_{[2]} & =\mathcal{K}_{[1]} .
\end{aligned}
$$

Notice that $H_{\alpha \dot{\alpha}}$ contributes only in $\mathcal{K}_{[2]}$. This is a consequence of its gauge transformation (8.1) which will remove any appropriate component. ${ }^{12}$ Also $\psi_{\alpha}$ contributes only in $\mathcal{K}_{[1]}$ because of its dimension. Similarly with type (2), all of these contributions have appropriate upper bounds which state that we can not have more than what it is provided by the superfields. Nevertheless component $H^{(2,2)}$ has a special status because no matter what it has to be present and that leads to the following condition

$$
\mathcal{K}_{(1,1)}^{H}+\mathcal{N}_{(1,1)}^{H}=1 .
$$

Putting everything together we are led to

$$
\mathcal{A}_{B}=6 \mathcal{N}_{(2,0)}+4 \mathcal{N}_{(1,1)}+\mathcal{N}_{(0,0)}+6 \mathcal{K} .
$$

Now, we can state the demand for matching of bosons and fermions

$$
6 \mathcal{N}_{(2,0)}+4 \mathcal{N}_{(1,1)}+\mathcal{N}_{(0,0)}+6 \mathcal{K}=6+8 \mathcal{N}_{\chi}
$$

The goal is to solve this Diophantine equation together with (8.4), (8.7) and (8.8). A solution will provide the answer to how many superfields we need to have $\left(\mathcal{N}_{\chi}^{*}, \mathcal{N}_{\psi}^{*}, \mathcal{N}_{V}^{*}\right)$ and the specific structure of the components. However, as we learned in the previous section, not all solutions are blindly accepted and we are interested in the most economical ones. If a solution results to the triplet $\left(\mathcal{N}_{\chi}^{*}, \mathcal{N}_{\psi}^{*}, \mathcal{N}_{V}^{*}\right)$ then we do not accept any other solution with $\left(\mathcal{N}_{\chi}^{*}+\delta_{\chi}, \mathcal{N}_{\psi}^{*}+\delta_{\psi}, \mathcal{N}_{V}^{*}+\delta_{V}\right)$ for some positive, integers $\delta_{\chi}, \delta_{\psi}, \delta_{V}$, because such a solution can be reduced to the original solution plus extra stuff. In this sense, we can distinguish among the irreducible descriptions and the non-irreducible ones. Our aim is to find all, different, irreducible, solutions.

\section{Solutions.}

(i) A good starting point for economical solutions is minimizing the degrees of free$\operatorname{dom}\left(12+8 \mathcal{N}_{\chi}\right)$. So let us start with $\mathcal{N}_{\chi}=0$. For that case, we must have $\mathcal{N}_{(2,0)}^{\chi}=\mathcal{N}_{(1,1)}^{\chi}=\mathcal{N}_{(0,0)}^{\chi}=\mathcal{K}_{(1,1)}^{\chi}=\mathcal{K}_{(2,0)}^{\chi}=k_{(1,1)}^{\chi}=k_{(2,0)}^{\chi}=k_{(1,1)}^{\psi}=k_{(1,1)}^{V}=0$ and we are led to the system of equations:

$$
\begin{aligned}
4 \mathcal{N}_{(1,1)}^{H}+\mathcal{N}_{V}+6 \mathcal{K}_{(1,1)}^{H} & =6, \\
\mathcal{K}_{(1,1)}^{H}+\mathcal{N}_{(1,1)}^{H} & =1, \\
\mathcal{K}_{(1,1)}^{H} & =k_{(2,0)}^{\psi} \leq \mathcal{N}_{\psi}, \\
0 & \leq \mathcal{N}_{(1,1)}^{H} \leq 1 .
\end{aligned}
$$

\footnotetext{
${ }^{12}$ Except the component $H^{(1,1)(A, A)}$ which is reserved to play the role of spin auxiliary component and participate in the spin dynamics.
} 
This system has exactly two solutions

$$
\begin{aligned}
& \text { a) } \mathcal{N}_{(1,1)}^{H}=0, \mathcal{N}_{V}=0, \mathcal{K}_{(1,1)}^{H}=k_{(2,0)}^{\psi}=1 \leq \mathcal{N}_{\psi} \text {, } \\
& \text { в) } \mathcal{N}_{(1,1)}^{H}=1, \mathcal{N}_{V}=2, \mathcal{K}_{(1,1)}^{H}=k_{(2,0)}^{\psi}=0 \leq \mathcal{N}_{\psi} \text {. }
\end{aligned}
$$

The first one corresponds to the triplet $\left(\mathcal{N}_{\chi}^{*}=0, \mathcal{N}_{\psi}^{*} \geq 1, \mathcal{N}_{V}^{*}=0\right)$ and as explained previously, it gives the "irreducible" configuration of $(0,1,0)$. This will turn out to be the $12 \times 12$, new-minimal formulation of supergravity supermultiplet with a superspace description based on $H_{\alpha \dot{\alpha}}$ and $\psi_{\alpha}$. The superfield $\psi_{\alpha},\left[\psi_{\alpha}\right]=-\frac{1}{2}$ that appears here is the prepotential of the real linear superfield $\left[U=\mathrm{D}^{\alpha} \overline{\mathrm{D}}^{2} \psi_{\alpha}+\overline{\mathrm{D}}^{\dot{\alpha}} \mathrm{D}^{2} \bar{\psi}_{\dot{\alpha}}\right]$ that is usually used to describe new-minimal supergravity. The off-shell auxiliary component spectrum has:

1. one real $(1,1)$-tensor of type $(1)$ :

$$
A_{\alpha \dot{\alpha}}, A_{\alpha \dot{\alpha}}=\bar{A}_{\alpha \dot{\alpha}}, \delta_{g} A_{\alpha \dot{\alpha}}=\partial_{\alpha \dot{\alpha}} \lambda, \lambda=\bar{\lambda},\left[A_{\alpha \dot{\alpha}}\right]=2,
$$

2. one (2,0)-tensor of type (1):

$$
\omega_{\alpha \beta}, \delta_{g} \omega_{\alpha \beta}=i \partial_{(\beta}{ }^{\dot{\alpha}} \ell_{\alpha) \dot{\alpha}}, \ell_{\alpha \dot{\alpha}}=\bar{\ell}_{\alpha \dot{\alpha}}, \ell_{\alpha \dot{\alpha}} \sim \ell_{\alpha \dot{\alpha}}+\partial_{\alpha \dot{\alpha}} \ell, \ell=\bar{\ell},\left[\omega_{\alpha \beta}\right]=1 .
$$

We can also see that by changing the auxiliary vector from real to imaginary, one can get a slightly different formulation of the supergravity supermultiplet which corresponds to new-new-minimal supergravity which is using a different real linear superfield $U=i\left(\mathrm{D}^{\alpha} \overline{\mathrm{D}}^{2} \psi_{\alpha}-\overline{\mathrm{D}}^{\dot{\alpha}} \mathrm{D}^{2} \bar{\psi}_{\dot{\alpha}}\right)$.

The second solution gives the irreducible triplet $(0,0,2)$. This corresponds to the $12 \times 12$, old minimal description of the supergravity supermultiplet based on $H_{\alpha \dot{\alpha}}$ and two real superfields $V_{1}, V_{2}$, which can be combined into a complex scalar superfield which is the prepotential of the chiral superfield $\left[\Phi=\overline{\mathrm{D}}^{2}\left(V_{1}+i V_{2}\right)\right]$ that is usually used in the old-minimal formulation. The off-shell auxiliary spectrum for this case is, as expected:

1. one real $(1,1)$-tensor of type $(2)$ :

$$
A_{\alpha \dot{\alpha}}, A_{\alpha \dot{\alpha}}=\bar{A}_{\alpha \dot{\alpha}}, \delta_{g} A_{\alpha \dot{\alpha}}=0,\left[A_{\alpha \dot{\alpha}}\right]=2,
$$

2. two real $(0,0)$-tensor of type $(2)$ :

$$
\begin{aligned}
& S, S=\bar{S}, \delta_{g} S=0,[S]=2, \\
& P, P=\bar{P}, \delta_{g} P=0,[P]=2 .
\end{aligned}
$$

(ii) Now, we consider solutions with $\mathcal{N}_{\chi} \neq 0$. Because we have already found solutions that correspond to the triplets $(0,1,0)$ and $(0,0,2)$, the solutions with $\mathcal{N}_{\chi} \neq 0$ in order to be acceptable as irreducible must have $\mathcal{N}_{\psi}<1 \Rightarrow \mathcal{N}_{\psi}=0$ and $\mathcal{N}_{V}<2$. For reasons of economy we do incremental steps so let's consider first the case of $\mathcal{N}_{\chi}=1$.

$$
6\left[\mathcal{N}_{(2,0)}^{\chi}+\mathcal{K}_{(2,0)}^{\chi}+\mathcal{K}_{(1,1)}^{\chi}\right]+4 \mathcal{N}_{(1,1)}^{\chi}+2 \mathcal{K}_{(1,1)}^{H}+\mathcal{N}_{(0,0)}^{\chi}+\mathcal{N}_{V}=10
$$


with

$$
\begin{aligned}
& 0 \leq \mathcal{N}_{(2,0)}^{\chi}+\mathcal{K}_{(2,0)}^{\chi} \leq 1, \\
& 0 \leq \mathcal{N}_{(1,1)}^{\chi}+\mathcal{K}_{(1,1)}^{\chi} \leq 2, \\
& 0 \leq \mathcal{N}_{(0,0)}^{\chi} \leq 2,0 \leq \mathcal{N}_{V} \leq 1, \\
& 0 \leq k_{(2,0)}^{\chi}=\mathcal{K}_{(1,1)}^{H}+\mathcal{K}_{(1,1)}^{\chi} \leq 1, \\
& 0 \leq \mathcal{K}_{(2,0)}^{\chi}=k_{(1,1)}^{\chi}+k_{(1,1)}^{V} \leq 1 .
\end{aligned}
$$

On top of the above, we have a set of conditional constraints coming from table 4 . In this case these constraints take the form:

1. If $\mathcal{N}_{(2,0)}^{\chi}+\mathcal{K}_{(2,0)}^{\chi}=0$, then $k_{(2,0)}^{\chi}=k_{(1,1)}^{\chi}=0$.

2. If $\mathcal{N}_{(1,1)}^{\chi}+\mathcal{K}_{(1,1)}^{\chi} \leq 1$, then $k_{(2,0)}^{\chi}=k_{(1,1)}^{\chi}=0$.

3. We can not have $\mathcal{N}_{(2,0)}^{\chi} \leq 1$ and $\mathcal{N}_{(1,1)}^{\chi}+\mathcal{K}_{(1,1)}^{\chi} \leq 1$ because there will be no candidate for the second fermionic auxiliary component needed to complete the pair.

Due to all the above constraints there is a unique solution of (8.15)

$$
\begin{aligned}
\mathcal{N}_{(0,0)}^{\chi}+\mathcal{N}_{V} & =2, \\
\mathcal{N}_{(2,0)}^{\chi}+\mathcal{K}_{(2,0)}^{\chi}+\mathcal{K}_{(1,1)}^{\chi} & =0 \rightarrow \mathcal{N}_{(2,0)}^{\chi}=\mathcal{K}_{(2,0)}^{\chi}=\mathcal{K}_{(1,1)}^{\chi}=k_{(2,0)}^{\chi}=k_{(1,1)}^{V}=0 \\
\mathcal{N}_{(1,1)}^{\chi} & =2 \\
\mathcal{K}_{(1,1)}^{H} & =0 .
\end{aligned}
$$

This solution stands for any value of $\mathcal{N}_{V}<2$, hence the irreducible piece will correspond to $\mathcal{N}_{V}=0$ giving the triplet $(1,0,0)$. This is the $20 \times 20$, non-minimal formulation of supergravity with a superspace description based on $H_{\alpha \dot{\alpha}}$ and $\chi_{\alpha}$ which is the prepotential of the complex linear superfield $\left[\Gamma=\overline{\mathrm{D}}^{\dot{\alpha}} \bar{\chi}_{\dot{\alpha}}\right]$ which traditionally used. The off-shell auxiliary spectrum of the theory has:

1. three real $(1,1)$-tensors of type $(2)$ :

$$
\begin{aligned}
& A_{\alpha \dot{\alpha}}, A_{\alpha \dot{\alpha}}=\bar{A}_{\alpha \dot{\alpha}}, \delta_{g} A_{\alpha \dot{\alpha}}=0,\left[A_{\alpha \dot{\alpha}}\right]=2, \\
& u_{\alpha \dot{\alpha}}, \quad u_{\alpha \dot{\alpha}}=\bar{u}_{\alpha \dot{\alpha}}, \quad \delta_{g} u_{\alpha \dot{\alpha}}=0,\left[u_{\alpha \dot{\alpha}}\right]=2, \\
& v_{\alpha \dot{\alpha}}, \quad v_{\alpha \dot{\alpha}}=\bar{v}_{\alpha \dot{\alpha}}, \quad \delta_{g} v_{\alpha \dot{\alpha}}=0,\left[v_{\alpha \dot{\alpha}}\right]=2,
\end{aligned}
$$

2. two real $(0,0)$-tensors of type $(2)$ :

$$
\begin{aligned}
& S, S=\bar{S}, \delta_{g} S=0,[S]=2, \\
& P, P=\bar{P}, \delta_{g} P=0,[P]=2,
\end{aligned}
$$

3. two $(1,0)$-tensors:

$$
\begin{aligned}
\beta_{\alpha}, \delta_{g} \beta_{\alpha} & =0,\left[\beta_{\alpha}\right]=\frac{5}{2}, \\
\rho_{\alpha}, \delta_{g} \rho_{\alpha} & =0,\left[\rho_{\alpha}\right]=\frac{3}{2} .
\end{aligned}
$$


4 - Based on the Diophantine equation we managed to classify and generate all possible irreducible formulation of supergravity supermultiplet. The answer was the familiar minimal (old and new) and non-minimal formulations. To complete the discussion, we have to find the gauge transformations for the superfields. Of course we know the gauge transformation of $H_{\alpha \dot{\alpha}}$ but we have to find it for $\chi_{\alpha}, \psi_{\alpha}$ and $V$ in a way that is consistent with the component spectrum.

For the old-minimal solution $(0,0,2)$, we combine the two real scalars $V_{1}, V_{2}$ into one complex superfield $W$ and we demand that its gauge transformation is such that it removes all its components (except of course $W^{(2,2)}$ which can not be removed). Looking through table 3, we find that in order to remove $W^{(1,2)(S)}$ we must have a $\mathrm{D}^{\alpha} J_{\alpha}$ term in the transformation with $J_{\alpha}$ unconstrained. Similarly in order to remove $W^{(2,1)(S)}$ we must have a $\overline{\mathrm{D}}^{\dot{\alpha}} I_{\dot{\alpha}}$ term in the transformation with $I_{\dot{\alpha}}$ unconstrained. Also, we observe that these two terms are enough to gauge away all other components. Furthermore, in order to use $H_{\alpha \dot{\alpha}}$ and $W$ to construct a non-trivial gauge invariant superspace theory we must have the set of gauge parameters of $W$ and the set of gauge parameters of $H_{\alpha \dot{\alpha}}$ to have an nonempty overlap. Hence $J_{\alpha}$ must be identified with $L_{\alpha}$. So the gauge transfornmation of $W$ is

$$
\delta_{g} W=\mathrm{D}^{\alpha} L_{\alpha}+\overline{\mathrm{D}}^{\dot{\alpha}} \Lambda_{\dot{\alpha}}
$$

for an arbitrary $\Lambda_{\dot{\alpha}}$. As we said previously, $W$ must be interpreted as the prepotential of the chiral superfield $\Phi\left[\Phi=\overline{\mathrm{D}}^{2} W\right]$ that is usually used. This is in agreement with (8.18) because it produces the correct transformation for $\Phi\left[\delta \Phi=\overline{\mathrm{D}}^{2} \mathrm{D}^{\alpha} L_{\alpha}\right]$.

For the new-minimal solution $(0,1,0)$, things become a little more interesting. The $\psi_{\alpha \beta}^{(1,2)(S)}$ component of superfield $\psi_{\alpha}$ must survive the gauge transformation because it generates the required $(2,0)$-tensor of type (1). In section 3.1 we showed that these type of components have a very special transformation. So the transformation of $\psi_{\alpha}$ must be choosen in a way that it respects these properties and also removes all other components. Going through table 3, we find that in order to remove components $\psi_{\alpha \dot{\alpha}}^{(2,1)(S)}$ we must have a term $\overline{\mathrm{D}}^{\dot{\alpha}} I_{\alpha \dot{\alpha}}$ in the transformation of $\psi_{\alpha}$, with $I_{\alpha \dot{\alpha}}$ unconstrained. Moreover, in order to remove component $\psi^{(1,2)(A)}$ we must have a term $\mathrm{D}_{\alpha} L$, with $L$ unconstrained. These two terms are enough to remove all other components of $\psi_{\alpha}$, but when we check the transformation of $\psi_{\alpha \beta}^{(1,2)(S)}$ we find that it does not have the correct properties. Specifically the gauge parameter, which in this case is $i\left[\mathrm{D}_{\alpha}, \overline{\mathrm{D}}_{\dot{\alpha}}\right] L \mid$ is not real. In order to make it real we must choose $L$ to be imaginary, but in return this choice conflicts with the gauge removal of $\psi^{(1,2)(A)}$. So it seems there is no consistent choice for the transformation of $\psi_{\alpha}$. However, even if we ignore this issue, we are still left with the fact that neither gauge parameters $\left(L, I_{\alpha \beta}\right)$ has the structure of the gauge parameter of $H_{\alpha \dot{\alpha}}$, so how can we use both these fields to construct an invariant superspace theory? Well, there is a way out that solves both issues at the same time and it is hidden in a subtle details.

In equation (8.7) we claimed that superfield $H_{\alpha \dot{\alpha}}$ does not contribute in the $\mathcal{K}_{[1]}$ terms, because we have used its gauge transformation to remove such terms. That means, we can now use the gauge parameter $L_{\alpha}$ and add it algebraically to the transformation of $\psi_{\alpha}$ 
without risking that it will remove the entire superfield. This allow us to choose $L$ imaginary, remove $\psi^{(1,2)(A)}$ and not remove $\psi_{\alpha \beta}^{(1,2)(S)}$ because the relevant component $L^{(1,2)(S)}$ has already been used to remove the component $H_{\alpha \beta}^{(1,1)(S, A)}$. So, the result is the gauge transformation of $\psi_{\alpha}$ must be

$$
\delta_{g} \psi_{\alpha}=L_{\alpha}+\mathrm{D}_{\alpha} K+\overline{\mathrm{D}}^{\dot{\alpha}} \Lambda_{\alpha \dot{\alpha}}, K=-\bar{K} .
$$

This also agrees with the interpretation of $\psi_{\alpha}$ as the prepotential of a real linear $U$, because it generates the known transformation for it $\delta U=\mathrm{D}^{\alpha} \overline{\mathrm{D}}^{2} L_{\alpha}+$ c.c.. $^{13}$

Finally, for the non-minimal solution $(1,0,0)$, we must have a tranformation that does not eliminate the components of $\chi_{\alpha \dot{\alpha}}^{(2,1)(S)}, \chi^{(1,2)(A)}$. Also we must have one $(1,0)$-tensor fermion with dimensions $\frac{3}{2}$ which must be there in order together with $\chi^{(2,2)}$ to complete the pair of auxiliary fermions. All other components must be removed by the gauge transformation. The removal of $\chi_{\alpha \beta}^{(1,2)(S)}$ forces us to have a term like $\mathrm{D}^{\beta} J_{\alpha \beta}$ in the transformation law of $\chi_{\alpha}$ with $J_{\alpha \beta}$ unconstrained. On the other hand, the removal of $\chi^{(1,0)(A)}$ forces us to introduce a term $\overline{\mathrm{D}}^{2} \Lambda_{\alpha}$ with arbitrary $\Lambda_{\alpha}$ or a term $\mathrm{D}_{\alpha} L$ with $L$ constrained such that it does not remove $\chi^{(1,2)(A)}$ or a term $\overline{\mathrm{D}}^{\dot{\alpha}} I_{\alpha \dot{\alpha}}$ with $I_{\alpha \dot{\alpha}}$ constrained such that it does not remove $\chi_{\alpha \dot{\alpha}}^{(2,1)(S)}$. At this point, the most general structure allowed is

$$
\delta_{g} \chi_{\alpha} \sim \mathrm{D}^{\beta} \Lambda_{\alpha \beta}+\overline{\mathrm{D}}^{2} \Lambda_{\alpha}+\mathrm{D}_{\alpha} \overline{\mathrm{D}}^{\dot{\alpha}} \bar{K}_{\dot{\alpha}}+\overline{\mathrm{D}}^{\dot{\alpha}} \mathrm{D}_{\alpha} \bar{I}_{\dot{\alpha}}
$$

with unconstrained parameters. The parameters $\Lambda_{\alpha}, K_{\alpha}, I_{\alpha}$ eventually have to be identified with the $L_{\alpha}$, which means the last term can be removed by a redefinition of $\chi_{\alpha}\left[\chi_{\alpha} \rightarrow\right.$ $\left.\chi_{\alpha}+\overline{\mathrm{D}}^{\dot{\alpha}} H_{\alpha \dot{\alpha}}\right]$. Also, there are two fermions left $\chi_{\alpha}^{(2,0)}, \chi_{\dot{\alpha}}^{(1,1)(A, S)}$ and we have to remove only one of them. We can either use the second term and remove the first fermion or use the third term and remove the first fermion. So the expression for the gauge transformation of $\chi_{\alpha}$ is:

$$
\delta_{g} \chi_{\alpha}=\overline{\mathrm{D}}^{2} L_{\alpha}+\frac{f}{2} \mathrm{D}_{\alpha} \overline{\mathrm{D}}^{\dot{\alpha}} \bar{L}_{\dot{\alpha}}+\mathrm{D}^{\beta} \Lambda_{\alpha \beta}
$$

where the parameter $f$ controls the relative coefficient between the first two terms. The interpretation of $\chi_{\alpha}$ as the prepotential of the complex linear $\Gamma$ compensator that is usually used, is in agreement with this result because it generates the correct transformation for it $\delta_{g} \Gamma=\overline{\mathrm{D}}^{\dot{\alpha}} \mathrm{D}^{2} \bar{L}_{\dot{\alpha}}+f \overline{\mathrm{D}}^{2} \mathrm{D}^{\alpha} L_{\alpha}$. However, we observe that if $f$ becomes very large then the second term in the transformation of $\Gamma$ dominates and $\delta \Gamma$ reduces to the transformation of the chiral in the first solution. Therefore, we want to impose the constraint $f \neq \infty$. Also, we can redefine $\Gamma$ to $\tilde{\Gamma}=\Gamma-f \overline{\mathrm{D}}^{\dot{\alpha}} \mathrm{D}^{\alpha} H_{\alpha \dot{\alpha}}$ and show that

$$
\delta_{g} \tilde{\Gamma}=(1-2 f) \overline{\mathrm{D}}^{\dot{\alpha}} \mathrm{D}^{2} \bar{L}_{\dot{\alpha}}-f \mathrm{D}^{\alpha} \overline{\mathrm{D}}^{2} L_{\alpha} .
$$

Hence, in order not to make contact with the second solution we must have $f \neq 1$ and $f \neq \frac{1}{3}$, thus giving as final result

$$
\delta_{g} \chi_{\alpha}=\overline{\mathrm{D}}^{2} L_{\alpha}+\frac{f}{2} \mathrm{D}_{\alpha} \overline{\mathrm{D}}^{\dot{\alpha}} \bar{L}_{\dot{\alpha}}+\mathrm{D}^{\beta} \Lambda_{\alpha \beta}, f \neq 1, \frac{1}{3}, \infty .
$$

\footnotetext{
${ }^{13}$ Very similar arguments will give rise to the gauge transformation law for the new-new-minimal description of supergravity supermultiplet, where $K$ is real.
} 
Having the set of superfields that we should use, their gauge transformation laws and knowing that the theory exist determines completely the superspace and component action for all formulations of a $Y=\frac{3}{2}$ theory.

This completes the application of this approach to the supergravity supermultiplet. We managed to show that it is possible to classify and derive the off-shell component spectrum from very basic requirements without constructing the action of the theory. This is the exact opposite of the mainstream approach where the spectrum of the theory is been derived from the details of an action.

\section{$9 \quad$ Arbitrary half-integer superspin}

We can continue applying successfully this method to even higher superspin theories. In this section, we will attempt to do so for the cases of arbitrary half-integer superspin theories. We will find that going beyond the supergravity multiplet introduces an interesting twist that can be exploited in order to successfully solve the Diophantine equation and obtain the answer.

1 - Consider the $Y=s+\frac{1}{2}$ multiplet. The dynamics of the theory will be described by one spin $s+1$ and one spin $s+\frac{1}{2}$. Therefore the off-shell degrees of freedom they provide are

$$
\begin{array}{rrr}
\operatorname{spin} s+1: & \left.\left(j^{2}+2\right)\right|_{j=s+1}=s^{2}+2 s+3, \\
\text { spin } s+\frac{1}{2}: & \left.\left(4 j^{2}+4 j+4\right)\right|_{j=s}=4 s^{2}+4 s+4
\end{array}
$$

and they will be described by the component fields $h_{\alpha(s+1) \dot{\alpha}(s+1)}, h_{\alpha(s-1) \dot{\alpha}(s-1)}, \psi_{\alpha(s+1) \dot{\alpha}(s)}$, $\psi_{\alpha(s) \dot{\alpha}(s-1)}$, and $\psi_{\alpha(s-1) \dot{\alpha}(s-2)}$ respectively together with appropriate gauge transformations.

2 - The highest spin is $s+1$, therefore the main superfield in the superspace description must be a real scalar superfield $H_{\alpha(s) \dot{\alpha}(s)}$. Following the same argument as in the previous case we can uniquely determine its gauge transformation to be

$$
\delta_{g} H_{\alpha(s) \dot{\alpha}(s)}=\frac{1}{s !} \mathrm{D}_{\left(\alpha_{s}\right.} \bar{L}_{\alpha(s-1)) \dot{\alpha}(s)}-\frac{1}{s !} \overline{\mathrm{D}}_{\left(\dot{\alpha}_{s}\right.} L_{\alpha(s) \dot{\alpha}(s-1))} .
$$

3 - Auxiliary superfields and matching bosons with fermions.

In order to find the list of appropriate auxiliary superfields, we can repeat the arguments we used in the previous sections, and list all superfields with total number of indices less than $2 s$. However for the general half-integer superspin supermultiplet there is a quicker approach which is based on a qualitative difference with the lower spin supermultiplets such as supergravity multiplet. As we can see from table 1 , the lowest rank fermion that $H_{\alpha(s) \dot{\alpha}(s)}$ provides is a $(s, s-1)$-tensor. Therefore $H_{\alpha(s) \dot{\alpha}(s)}$ can not generate the $\psi_{\alpha(s-1) \dot{\alpha}(s-2)}$ component that is required for the off-shell description of spin $s+\frac{1}{2}$. For the case of supergravity multiplet, this issue was avoided because this component is not relevant for $s \leq 1$. So we know immediately, without checking the matching of bosons with fermions, that we need auxiliary superfields and unlike the previous cases, they have to provide not only auxiliary fields but also the missing dynamical component. The most economical way to generate this missing component without introducing any other dynamical components with even 
lower rank is to consider auxiliary superfields $A_{\alpha(k) \dot{\alpha}(l)}$ such that their lowest rank component $A_{\alpha(k-1) \dot{\alpha}(l-1)}^{(1,1)(A, A)}$ can be identified with $\psi_{\alpha(s-1) \dot{\alpha}(s-2)}$. This would suggest to consider the superfield

$$
k-1=s-1, l-1=s-2, d+1=\frac{3}{2} \quad \Rightarrow \quad \chi_{\alpha(s) \dot{\alpha}(s-1)}, d_{\chi}=\frac{1}{2} .
$$

However, we see in table 2 that there is a possibility for component $A_{\alpha(k-1) \dot{\alpha}(l-1)}^{(1,1)(A, A)}$ to be gauged away by an appropriate gauge transformation. In that case, we go to the next lowest rank tensors within the same group. These are the components $A_{\alpha(k+1) \dot{\alpha}(l-1)}^{(1,1)(S, A)}$, $A_{\alpha(k-1) \dot{\alpha}(l+1)}^{(1,1)(A, S)}, A_{\alpha(k) \dot{\alpha}(l)}^{(2,0)}, A_{\alpha(k) \dot{\alpha}(l)}^{(0,2)}{ }^{14}$ So, we must add to the list of potential auxiliary superfields $\chi_{\alpha(s-1) \dot{\alpha}(s-2)}, \chi_{\alpha(s-2) \dot{\alpha}(s-3)}$. This is a huge shortcut because instead of considering the entire list of potential superfields $\left\{A_{\alpha(s) \dot{\alpha}(s-1)}, \ldots, A\right\}$ we only have to consider $\left\{\chi_{\alpha(s) \dot{\alpha}(s-1)}, \chi_{\alpha(s-1) \dot{\alpha}(s-2)}, \chi_{\alpha(s) \dot{\alpha}(s-3)}\right\}$. Now, we analyze the set of potential auxiliary components these superfields introduce. First of all, due to the index structure all of the auxiliary bosons must be of type (2). Secondly, all of these superfields introduce auxiliary fermions. We obtain:

\begin{tabular}{|c|c|c|c|c|c|}
\hline $1 \times H_{\alpha(s) \dot{\alpha}(s)}:$ & Bosons: & $\begin{array}{l}\text { Tensor } \\
(s, s)\end{array}$ & $\begin{array}{c}\text { Dimensions } \\
{[2]}\end{array}$ & $\begin{array}{l}\text { off-shell d.o.f. } \\
(s+1)^{2}\end{array}$ & $\begin{array}{c}\text { multiplicity } \\
1\end{array}$ \\
\hline \multirow[t]{2}{*}{$\mathcal{N}_{1} \times \chi_{\alpha(s) \dot{\alpha}(s-1)}$} & Fermions: & $(s, s-1)$ & {$\left[\frac{5}{2}\right]$} & $2(s+1) s$ & $\mathcal{N}_{1}$ \\
\hline & Bosons: & $\begin{array}{l}(s+1, s-1) \\
(s-1, s-1) \\
(s, s) \\
(s, s-2)\end{array}$ & $\begin{array}{l}{[2]} \\
{[2]} \\
{[2]} \\
{[2]}\end{array}$ & $\begin{array}{l}2(s+2) s \\
s^{2} \\
(s+1)^{2} \\
2(s+1)(s-1)\end{array}$ & $\begin{array}{l}\leq \mathcal{N}_{1} \\
\leq 2 \mathcal{N}_{1} \\
\leq 2 \mathcal{N}_{1} \\
\leq \mathcal{N}_{1}\end{array}$ \\
\hline \multirow[t]{2}{*}{$\mathcal{N}_{2} \times \chi_{\alpha(s-1) \dot{\alpha}(s-2)}$} & Fermions: & $(s-1, s-2)$ & {$\left[\frac{5}{2}\right]$} & $2 s(s-1)$ & $\mathcal{N}_{2}$ \\
\hline & Bosons: & $\begin{array}{l}(s, s-2) \\
(s-2, s-2) \\
(s-1, s-1) \\
(s-1, s-3)\end{array}$ & $\begin{array}{l}{[2]} \\
{[2]} \\
{[2]} \\
{[2]}\end{array}$ & $\begin{array}{l}2(s+1)(s-1) \\
(s-1)^{2} \\
s^{2} \\
2 s(s-2)\end{array}$ & $\begin{array}{l}\leq \mathcal{N}_{2} \\
\leq 2 \mathcal{N}_{2} \\
\leq 2 \mathcal{N}_{2} \\
\leq \mathcal{N}_{2}\end{array}$ \\
\hline \multirow[t]{2}{*}{$\mathcal{N}_{3} \times \chi_{\alpha(s) \dot{\alpha}(s-3)}$} & Fermions: & $(s, s-3)$ & {$\left[\frac{5}{2}\right]$} & $2(s+1)(s-2)$ & $\mathcal{N}_{3}$ \\
\hline & Bosons: & $\begin{array}{l}(s+1, s-3) \\
(s-1, s-3) \\
(s, s-2) \\
(s, s-4)\end{array}$ & $\begin{array}{l}{[2]} \\
{[2]} \\
{[2]} \\
{[2]}\end{array}$ & $\begin{array}{l}2(s+2)(s-2) \\
2 s(s-2) \\
2(s+1)(s-1) \\
2(s+1)(s-3)\end{array}$ & $\begin{array}{l}\leq \mathcal{N}_{3} \\
\leq \mathcal{N}_{3} \\
\leq \mathcal{N}_{3} \\
\leq \mathcal{N}_{3}\end{array}$ \\
\hline
\end{tabular}

Therefore the auxiliary components coming from $H$ and the $\chi \mathrm{s}$ are

$$
\begin{aligned}
\mathcal{A}_{B}= & (s+1)^{2}+2(s+2) s \mathcal{N}_{(s+1, s-1)}+2(s+1)(s-1) \mathcal{N}_{(s, s-2)}+2 s(s-2) \mathcal{N}_{(s-1, s-3)} \\
& +2(s+2)(s-2) \mathcal{N}_{(s+1, s-3)}+2(s+1)(s-3) \mathcal{N}_{(s, s-4)} \\
& +(s+1)^{2} \mathcal{N}_{(s, s)}+s^{2} \mathcal{N}_{(s-1, s-1)}+(s-1)^{2} \mathcal{N}_{(s-2, s-2)} \\
\mathcal{A}_{F}= & 4(s+1) s \mathcal{N}_{1}+4 s(s-1) \mathcal{N}_{2}+4(s+1)(s-2) \mathcal{N}_{3}
\end{aligned}
$$

\footnotetext{
${ }^{14}$ If $A_{\alpha(k-1) \dot{\alpha}(l-1)}^{(1,1)(A, A)}$ can be removed then so can $A_{\alpha(k) \dot{\alpha}(l)}^{(2,0)}$ or $A_{\alpha(k) \dot{\alpha}(l)}^{(0,2)}$.
} 
where

$$
\begin{aligned}
\mathcal{N}_{(s+1, s-1)} & =N_{(s+1, s-1)}^{1}, & N_{(s+1, s-1)}^{1} & \leq \mathcal{N}_{1} \\
\mathcal{N}_{(s, s-2)} & =N_{(s, s-2)}^{1}+N_{(s, s-2)}^{2}+N_{(s, s-2)}^{3}, & N_{(s, s-2)}^{1} & \leq \mathcal{N}_{1}, \\
\mathcal{N}_{(s-1, s-3)} & =N_{(s-1, s-3)}^{2}+N_{(s-1, s-3)}^{3}, & N_{(s-1, s-3)}^{2} & \leq \mathcal{N}_{2}, \quad N_{(s-1, s-3)}^{3} \leq \mathcal{N}_{3}, \\
\mathcal{N}_{(s+1, s-3)} & =N_{(s+1, s-3)}^{3}, & N_{(s+1, s-3)} & \leq \mathcal{N}_{3}, \\
\mathcal{N}_{(s, s-4)} & =N_{(s, s-4)}^{3}, & N_{(s, s-4)}^{3} & \leq \mathcal{N}_{3}, \\
\mathcal{N}_{(s, s)} & =N_{(s, s)}^{1}, & N_{(s, s)}^{1} & \leq 2 \mathcal{N}_{1}, \\
\mathcal{N}_{(s-1, s-1)} & =N_{(s-1, s-1)}^{1}+N_{(s-1, s-1)}^{2}, & N_{(s-1, s-1)} & \leq 2 \mathcal{N}_{1}, \quad N_{(s-1, s-1)} \leq 2 \mathcal{N}_{2}, \\
\mathcal{N}_{(s-2, s-2)} & =N_{(s-2, s-2)}^{2}, & N_{(s-2, s-2)}^{2} & \leq 2 \mathcal{N}_{2} .
\end{aligned}
$$

The matching of the bosonic and fermionic degrees of freedom condition is

$$
\underbrace{s^{2}+2 s+3}_{\text {spin } s+1}+\mathcal{A}_{B}=\underbrace{4 s^{2}+4 s+4}_{\text {spin } s+\frac{1}{2}}+\mathcal{A}_{F} \Rightarrow \quad \mathcal{A}_{B}=2 s^{2}+(s+1)^{2}+\mathcal{A}_{F} .
$$

This is the Diophantine equation we have to solve and the acceptable solutions will determine the spectrum of the higher spin supersymmetric theories. The brute force method of solving it is to interpret (9.5) as a polynomial equation which must hold for every value of $s \geq 2$ and therefore the coefficients for each power of $s$ in both sides must match. However, there is a more elegant method. First of all, notice that the $(s+1)^{2}$ term in the right hand side of the above equation will cancel exactly the $(s+1)^{2}$ term in $\mathcal{A}_{B}$, coming from the auxiliary d.o.f of superfield $H_{\alpha(s) \dot{\alpha}(s)}$. The rest of the coefficients in $\mathcal{A}_{B}$ have the structure $(k)^{2}$ or $k(k-2)$ or $k(k-4)$ for some integers $k$. Similarly, notice that the numerical coefficients in $\mathcal{A}_{F}$ are of the form $(k+1) k$ or $(k+2) k$ for some integer $k$. So solving (9.5) means that we must be able to convert from one structure to the other. This is indeed possible because of the following three identities

$$
\begin{aligned}
& \text { (I) } 4(k+m) k=2(k+m)^{2}+2(k+m)(k-m), \\
& \text { (II) } 4(k+m) k=2 k^{2}+2(k+2 m) k \text {, } \\
& \text { (III) } 4(k+m) k=6 k^{2}-2 k(k-2 m) .
\end{aligned}
$$

Using them, we convert all the terms that appear in $\mathcal{A}_{F}$ :

$$
\begin{aligned}
4(s+1) s \mathcal{N}_{1}= & \begin{cases}2(s+1)^{2} \mathcal{N}_{1}+2(s+1)(s-1) \mathcal{N}_{1}, & (I) \\
2 s^{2} \mathcal{N}_{1}+2(s+2) s \mathcal{N}_{1}, & (I I) \\
6 s^{2} \mathcal{N}_{1}-2 s(s-2) \mathcal{N}_{1}, & (I I I)\end{cases} \\
4 s(s-1) \mathcal{N}_{2}= & \begin{cases}2 s^{2} \mathcal{N}_{2}+2 s(s-2) \mathcal{N}_{2}, & (I) \\
2(s-1)^{2} \mathcal{N}_{2}+2(s+1)(s-1) \mathcal{N}_{2}, & (I I) \\
6(s-1)^{2} \mathcal{N}_{2}-2(s-1)(s-3) \mathcal{N}_{2}, & (I I I)\end{cases} \\
4(s+1)(s-2) \mathcal{N}_{3}= & \begin{cases}2(s+1)^{2} \mathcal{N}_{3}+2(s+1)(s-5) \mathcal{N}_{3}, & (I) \\
2(s-2)^{2} \mathcal{N}_{3}+2(s+4)(s-2) \mathcal{N}_{3}, & (I I) \\
6(s-2)^{2} \mathcal{N}_{3}-2(s-2)(s-8) \mathcal{N}_{3}, & (I I I)\end{cases}
\end{aligned}
$$


From the (9.11) we can easily see that $\mathcal{N}_{3}$ has to be zero, because non of the three identities can generate terms that appear in $\mathcal{A}_{B}$ and from (9.9), (9.10) we deduce that only identities $(I)$ and $(I I)$ are relevant. Applying $(I)$ in (9.5) we get a solution with $\mathcal{N}_{2}=\mathcal{N}_{3}=0, \mathcal{N}_{1}=1$ :

$$
\begin{aligned}
N_{(\ldots)}^{2}=0, & N_{(s+1, s-1)}^{1}=0, \\
N_{(\ldots)}^{3}=0, \quad N_{(s-1, s-1)}^{1} & =2, \\
N_{(s, s)}^{1} & =2, \\
N_{(s, s-2)}^{1} & =1 .
\end{aligned}
$$

Using $(I I)$ we get a solution with $\mathcal{N}_{1}=\mathcal{N}_{3}=0, \quad \mathcal{N}_{2}=1$ :

$$
\begin{aligned}
N_{(\ldots)}^{1}=0, & N_{(s-1, s-1)}^{2}=2, \\
N_{(\ldots)}^{3}=0, \quad N_{(s, s-2)}^{2} & =1, \\
N_{(s-2, s-2)}^{2} & =2, \\
N_{(s-1, s-3)}^{2} & =0 .
\end{aligned}
$$

The result is that there are two different formulation of the theory because there are exactly two ways that we can convert the structure of the fermionic d.o.f. to bosonic d.o.f. For the first solution, the theory has $8 s^{2}+8 s+4 \times 8 s^{2}+8 s+4$ d.o.f., its superspace description is based on the superfields $H_{\alpha(s) \dot{\alpha}(s)}$ and $\chi_{\alpha(s) \dot{\alpha}(s-1)}$ and the off-shell auxiliary component spectrum has:

1. three real $(s, s)$-tensors:

$$
\begin{array}{lll}
A_{\alpha(s) \dot{\alpha}(s)}, & A_{\alpha(s) \dot{\alpha}(s)}=\bar{A}_{\alpha(s) \dot{\alpha}(s)}, \delta_{g} A_{\alpha(s) \dot{\alpha}(s)}=0,\left[A_{\alpha(s) \dot{\alpha}(s)}\right]=2, \\
u_{\alpha(s) \dot{\alpha}(s)}, & u_{\alpha(s) \dot{\alpha}(s)}=\bar{u}_{\alpha(s) \dot{\alpha}(s)}, & \delta_{g} u_{\alpha(s) \dot{\alpha}(s)}=0,\left[u_{\alpha(s) \dot{\alpha}(s)}\right]=2, \\
v_{\alpha(s) \dot{\alpha}(s)}, & v_{\alpha(s) \dot{\alpha}(s)}=\bar{v}_{\alpha(s) \dot{\alpha}(s)}, & \delta_{g} v_{\alpha(s) \dot{\alpha}(s)}=0,\left[v_{\alpha(s) \dot{\alpha}(s)}\right]=2,
\end{array}
$$

2. two real $(s-1, s-1)$-tensors:

$$
\begin{aligned}
& S_{\alpha(s-1) \dot{\alpha}(s-1)}, S_{\alpha(s-1) \dot{\alpha}(s-1)}=\bar{S}_{\alpha(s-1) \dot{\alpha}(s-1)}, \delta_{g} S_{\alpha(s-1) \dot{\alpha}(s-1)}=0,\left[S_{\alpha(s-1) \dot{\alpha}(s-1)}\right]=2, \\
& P_{\alpha(s-1) \dot{\alpha}(s-1)}, P_{\alpha(s-1) \dot{\alpha}(s-1)}=\bar{P}_{\alpha(s-1) \dot{\alpha}(s-1)}, \delta_{g} P_{\alpha(s-1) \dot{\alpha}(s-1)}=0,\left[P_{\alpha(s-1) \dot{\alpha}(s-1)}\right]=2,
\end{aligned}
$$

3. one $(s, s-2)$-tensor:

$$
U_{\alpha(s) \dot{\alpha}(s-2)}, \delta_{g} U_{\alpha(s) \dot{\alpha}(s-2)}=0,\left[U_{\alpha(s) \dot{\alpha}(s-2)}\right]=2,
$$

4. two $(s, s-1)$-tensors:

$$
\begin{aligned}
& \beta_{\alpha(s) \dot{\alpha}(s-1)}, \delta_{g} \beta_{\alpha(s) \dot{\alpha}(s-1)}=0,\left[\beta_{\alpha(s) \dot{\alpha}(s-1)}\right]=\frac{5}{2}, \\
& \rho_{\alpha(s) \dot{\alpha}(s-1)}, \delta_{g} \rho_{\alpha(s) \dot{\alpha}(s-1)}=0,\left[\rho_{\alpha(s) \dot{\alpha}(s-1)}\right]=\frac{3}{2} .
\end{aligned}
$$

The second solution corresponds to a formulation with $8 s^{2}+4 \times 8 s^{2}+4$ d.o.f. based on the superfields $H_{\alpha(s) \dot{\alpha}(s)}, \chi_{\alpha(s-1) \dot{\alpha}(s-2)}$. The off-shell auxiliary spectrum has: 
1. one real $(s, s)$-tensor:

$$
A_{\alpha(s) \dot{\alpha}(s)}, A_{\alpha(s) \dot{\alpha}(s)}=\bar{A}_{\alpha(s) \dot{\alpha}(s)}, \delta_{g} A_{\alpha(s) \dot{\alpha}(s)}=0,\left[A_{\alpha(s) \dot{\alpha}(s)}\right]=2,
$$

2. two real $(s-1, s-1)$-tensors:

$$
\begin{aligned}
& u_{\alpha(s-1) \dot{\alpha}(s-1)}, u_{\alpha(s-1) \dot{\alpha}(s-1)}=\bar{u}_{\alpha(s-1) \dot{\alpha}(s-1)}, \delta_{g} u_{\alpha(s-1) \dot{\alpha}(s-1)}=0,\left[u_{\alpha(s-1) \dot{\alpha}(s-1)}\right]=2, \\
& v_{\alpha(s-1) \dot{\alpha}(s-1)}, v_{\alpha(s-1) \dot{\alpha}(s-1)}=\bar{v}_{\alpha(s-1) \dot{\alpha}(s-1)}, \delta_{g} v_{\alpha(s-1) \dot{\alpha}(s-1)}=0,\left[v_{\alpha(s-1) \dot{\alpha}(s-1)}\right]=2,
\end{aligned}
$$

3. two real $(s-2, s-2)$-tensors

$$
\begin{aligned}
& S_{\alpha(s-1) \dot{\alpha}(s-1)}, S_{\alpha(s-1) \dot{\alpha}(s-1)}=\bar{S}_{\alpha(s-1) \dot{\alpha}(s-1)}, \delta_{g} S_{\alpha(s-1) \dot{\alpha}(s-1)}=0,\left[S_{\alpha(s-1) \dot{\alpha}(s-1)}\right]=2 \\
& P_{\alpha(s-1) \dot{\alpha}(s-1)}, P_{\alpha(s-1) \dot{\alpha}(s-1)}=\bar{P}_{\alpha(s-1) \dot{\alpha}(s-1)}, \delta_{g} P_{\alpha(s-1) \dot{\alpha}(s-1)}=0,\left[P_{\alpha(s-1) \dot{\alpha}(s-1)}\right]=2
\end{aligned}
$$

4. one $(s, s-2)$-tensor:

$$
U_{\alpha(s) \dot{\alpha}(s-2)}, \delta_{g} U_{\alpha(s) \dot{\alpha}(s-2)}=0,\left[U_{\alpha(s) \dot{\alpha}(s-2)}\right]=2,
$$

5. two $(s, s-1)$-tensors:

$$
\begin{aligned}
& \beta_{\alpha(s) \dot{\alpha}(s-1)}, \delta_{g} \beta_{\alpha(s) \dot{\alpha}(s-1)}=0,\left[\beta_{\alpha(s) \dot{\alpha}(s-1)}\right]=\frac{5}{2}, \\
& \rho_{\alpha(s) \dot{\alpha}(s-1)}, \delta_{g} \rho_{\alpha(s) \dot{\alpha}(s-1)}=0,\left[\rho_{\alpha(s) \dot{\alpha}(s-1)}\right]=\frac{3}{2} .
\end{aligned}
$$

4 - Gauge transformations.

Having the detailed spectrum for each theory, we can deduce the gauge transformations for each superfield. For the first solution, the components $\chi^{(1,0)(S)}, \chi^{(1,0)(A)}, \chi^{(1,2)(S)}$, $\chi^{(1,1)(S, S)}, \chi^{(1,1)(S, A)}$ must be gauged away while the components $\chi^{(1,2)(A)}, \chi^{(2,1)(S)}$, $\chi^{(2,1)(A)}, \chi^{(1,1)(A, A)}$ must not be removed. Using tables 2,3 and demand a non zero overlap with the gauge parameter of $H_{\alpha(s) \dot{\alpha}(s)}$ we deduce that

$$
\delta_{g} \chi_{\alpha(s) \dot{\alpha}(s-1)}=\overline{\mathrm{D}}^{2} L_{\alpha(s) \dot{\alpha}(s-1)}+\mathrm{D}^{\beta} \Lambda_{\beta \alpha(s) \dot{\alpha}(s-1)} .
$$

Up to overall redefinitions of $\chi$ this is exactly the transformation of the compensator in [3] and therefore it will lead to the construction of the same superspace action. For the second solution, following similar arguments one can find the proper transformation for $\chi_{\alpha(s-1) \dot{\alpha}(s-2)}$ and it is in agreement with the results in [3].

\section{Arbitrary integer superspin}

So far, we have worked out in detail the application of the method to the half-integer superspin theories. In this section we will appy it to arbitrary integer superspin $Y=s$. 
1 - The dynamics of the theory will be described by one spin $s$ and one spin $s+\frac{1}{2}$. Therefore the off-shell degrees of freedom they provide are

$$
\begin{aligned}
\operatorname{spin} s: & \left.\left(j^{2}+2\right)\right|_{j=s}=s^{2}+2, \\
\operatorname{spin} s+\frac{1}{2}: & \left.\left(4 j^{2}+4 j+4\right)\right|_{j=s}=4 s^{2}+4 s+4
\end{aligned}
$$

and they will be described by the components $h_{\alpha(s) \dot{\alpha}(s)}, h_{\alpha(s-2) \dot{\alpha}(s-2)}, \psi_{\alpha(s+1) \dot{\alpha}(s)}$, $\psi_{\alpha(s) \dot{\alpha}(s-1)}, \psi_{\alpha(s-1) \dot{\alpha}(s-2)}$ respectively together with appropriate gauge transformations.

2 - The highest spin is $s+1 / 2$, therefore the main superfield in the superspace description must $\Psi_{\alpha(s) \dot{\alpha}(s-1)}$ with engineering dimensions of $\frac{1}{2}$.

3 - Auxiliary superfields.

Similar to the arbitrary half-integer case, the main superfield can not generate all components required for the off-shell description of the spins. In this case, the superfield $\Psi_{\alpha(s) \dot{\alpha}(s-1)}$ does not include a $(s-2 s-2)$-tensor to play the role of $h_{\alpha(s-2) \dot{\alpha}(s-2)}$. Following the arguments of the previous section, the list of auxiliary superfields we must consider is $\left\{V_{\alpha(s-1) \dot{\alpha}(s-1)}, Z_{\alpha(s-2) \dot{\alpha}(s-2)}, W_{\alpha(s-1) \dot{\alpha}(s-3)}\right\}$. However, the component $h_{\alpha(s-2) \dot{\alpha}(s-2)}$ has to be real and therefore it is obvious that only one of these choices can give rise to a real component of this type and that is a real superfield $V_{\alpha(s-1) \dot{\alpha}(s-1)}$. Nevertheless, for the sake of thoroughness we will entertain the possibility of other options and we will see that the Diophantine equation will reject them.

So let us assume that we have $\mathcal{N}_{1}$ copies of a real $(s-1, s-1)$ superfield, $\mathcal{N}_{2}$ copies of a real $(s-2, s-2)$ superfield and $\mathcal{N}_{3}$ copies of a $(s-1, s-3)$ superfield. These provide the following set of potential auxiliary components ${ }^{15}$

\begin{tabular}{|cclclc|}
\hline \multirow{2}{*}{$1 \times \Psi_{\alpha(s) \dot{\alpha}(s-1)}:$} & & Tensor & Dimensions & off-shell d.o.f. & multiplicity \\
& Fermions: & $(s, s-1)$ & {$\left[\frac{5}{2}\right]$} & $2(s+1) s$ & 1 \\
& Bosons: & $(s+1, s-1)$ & {$[2]$} & $2(s+2) s$ & $\leq 1$ \\
& & $(s-1, s-1)$ & {$[2]$} & $s^{2}$ & $\leq 2$ \\
& & $(s, s)$ & {$[2]$} & $(s+1)^{2}$ & $\leq 2$ \\
& & $(s, s-2)$ & {$[2]$} & $2(s+1)(s-1)$ & $\leq 1$ \\
\hline $\mathcal{N}_{1} \times V_{\alpha(s-1) \dot{\alpha}(s-1)}:$ & Bosons: & $(s-1, s-1)$ & {$[2]$} & $s^{2}$ & $\mathcal{N}_{1}$ \\
\hline $\mathcal{N}_{2} \times Z_{\alpha(s-2) \dot{\alpha}(s-2)}:$ & Bosons: & $(s-2, s-2)$ & {$[2]$} & $(s-1)^{2}$ & $\mathcal{N}_{2}$ \\
\hline $\mathcal{N}_{3} \times W_{\alpha(s-1) \dot{\alpha}(s-3)}:$ & Bosons: & $(s-1, s-3)$ & {$[2]$} & $2 s(s-2)$ & $\mathcal{N}_{3}$ \\
\hline
\end{tabular}

and the Diophantine equation that balances the bosons with fermions is

$$
\begin{aligned}
& 2(s+2) s \mathcal{N}_{(s+1, s-1)}^{\Psi}+2(s+1) s \mathcal{N}_{(s, s-2)}^{\Psi}+2 s(s-2) \mathcal{N}_{3} \\
& \quad+(s+1)^{2} \mathcal{N}_{(s, s)}^{\Psi}+s^{2}\left[\mathcal{N}_{1}+\mathcal{N}_{(s-1, s-1)}^{\Psi}\right]+(s-1)^{2} \mathcal{N}_{2}=7(s+1) s+(s+2)
\end{aligned}
$$

\footnotetext{
${ }^{15}$ Because all of the superfields are bosonic there are no auxiliary fermions and because we are in the case of arbitrary $s$ all the auxiliary bosons must be of type (2).
} 
where $\mathcal{N}_{(s+1, s-1)}^{\Psi} \leq 1, \mathcal{N}_{(s, s-2)}^{\Psi} \leq 1, \mathcal{N}_{(s, s)}^{\Psi} \leq 2, \mathcal{N}_{(s-1, s-1)}^{\Psi} \leq 2$. To solve this equation we can repeat the steps we did in the previous section, using (9.6). Doing that, we quickly find that only identity $(I I)$ is relevant in this case, hence there is only one economical solution. However, in order to demonstrate the polynomial approach that was mentioned previously, we will follow the latter in this section. In the polynomial approach, we equate the coefficients of different powers of $s$ between the left and right hand sided of the equation. We get:

$$
\begin{array}{r}
2 \mathcal{N}_{(s+1, s-1)}^{\Psi}+2 \mathcal{N}_{(s, s-2)}^{\Psi}+\mathcal{N}_{(s, s)}^{\Psi}+\mathcal{N}_{(s-1, s-1)}^{\Psi}+\mathcal{N}_{1}+\mathcal{N}_{2}+2 \mathcal{N}_{3}=7 \\
4 \mathcal{N}_{(s+1, s-1)}^{\Psi}+2 \mathcal{N}_{(s, s-2)}^{\Psi}+2 \mathcal{N}_{(s, s)}^{\Psi}-2 \mathcal{N}_{2}-4 \mathcal{N}_{3}=8 \\
\mathcal{N}_{(s, s)}^{\Psi}+\mathcal{N}_{2}=2 .
\end{array}
$$

Taking into account the upper bound constraints for the various parameters, we find a unique economical solution that corresponds to:

$$
\begin{aligned}
& \mathcal{N}_{2}=0, \mathcal{N}_{(s+1, s-1)}^{\Psi}=1, \\
& \mathcal{N}_{3}=0, \mathcal{N}_{(s-1, s-1)}^{\Psi}=2, \\
& \mathcal{N}_{1}=1, \mathcal{N}_{(s, s)}^{\Psi}=2, \\
& \mathcal{N}_{(s, s-2)}^{\Psi}=0,
\end{aligned}
$$

which confirms our suggestion based on the reality of the missing $(s-2, s-2)$-tensor component. The conclusion is that for arbitrary integer superspin theories there is a unique formulation of the theory based on a $(s, s-1)$ superfield and a real $(s-1, s-1)$ superfield with $8 s^{2}+8 s+4 \times 8 s^{2}+8 s+4$ components. The off-shell auxiliary component spectrum of it has:

1. two real $(s, s)$-tensors:

$$
\begin{aligned}
& u_{\alpha(s) \dot{\alpha}(s)}, u_{\alpha(s) \dot{\alpha}(s)}=\bar{u}_{\alpha(s) \dot{\alpha}(s)}, \delta_{g} u_{\alpha(s) \dot{\alpha}(s)}=0,\left[u_{\alpha(s) \dot{\alpha}(s)}\right]=2, \\
& v_{\alpha(s) \dot{\alpha}(s)}, v_{\alpha(s) \dot{\alpha}(s)}=\bar{v}_{\alpha(s) \dot{\alpha}(s)}, \delta_{g} v_{\alpha(s) \dot{\alpha}(s)}=0,\left[v_{\alpha(s) \dot{\alpha}(s)}\right]=2,
\end{aligned}
$$

2. three real $(s-1, s-1)$-tensors:

$$
\begin{aligned}
& A_{\alpha(s-1) \dot{\alpha}(s-1)}, A_{\alpha(s-1) \dot{\alpha}(s-1)}=\bar{A}_{\alpha(s-1) \dot{\alpha}(s-1)}, \delta_{g} A_{\alpha(s-1) \dot{\alpha}(s-1)}=0,\left[A_{\alpha(s-1) \dot{\alpha}(s-1)}\right]=2, \\
& S_{\alpha(s-1) \dot{\alpha}(s-1)}, S_{\alpha(s-1) \dot{\alpha}(s-1)}=\bar{S}_{\alpha(s-1) \dot{\alpha}(s-1)}, \delta_{g} S_{\alpha(s-1) \dot{\alpha}(s-1)}=0,\left[S_{\alpha(s-1) \dot{\alpha}(s-1)}\right]=2, \\
& P_{\alpha(s-1) \dot{\alpha}(s-1)}, P_{\alpha(s-1) \dot{\alpha}(s-1)}=\bar{P}_{\alpha(s-1) \dot{\alpha}(s-1)}, \delta_{g} P_{\alpha(s-1) \dot{\alpha}(s-1)}=0,\left[P_{\alpha(s-1) \dot{\alpha}(s-1)}\right]=2,
\end{aligned}
$$

3. one $(s+1, s-1)$-tensor:

$$
U_{\alpha(s+1) \dot{\alpha}(s-1)}, \delta_{g} U_{\alpha(s+1) \dot{\alpha}(s-1)}=0,\left[U_{\alpha(s+1) \dot{\alpha}(s-1)}\right]=2 .
$$

4 - To find the gauge transformation we need to have for the superfields $\Psi_{\alpha(s) \dot{\alpha}(s-1)}$ and $V_{\alpha(s-1) \dot{\alpha}(s-1)}$, we go through the list of components that must be removed (like $\left.\Psi_{\alpha(s) \dot{\alpha}(s-2)}^{(2,1)}\right)$ and via tables 2 and 3 select the appropriate transformation that does it while preserving 
the components that must survive (like $V_{\alpha(s-2) \dot{\alpha}(s-2)}^{(1,1)(A, A)}$ ). It is straight forward to find that the transformations we must have are:

$$
\begin{aligned}
\delta_{g} \Psi_{\alpha(s) \dot{\alpha}(s-1)} & =\mathrm{D}^{2} L_{\alpha(s) \dot{\alpha}(s-1)}+\frac{1}{(s-1) !} \overline{\mathrm{D}}_{\left(\dot{\alpha}_{s-1}\right.} \Lambda_{\alpha(s) \dot{\alpha}(s-2))}, \\
\delta_{g} V_{\alpha(s-1) \dot{\alpha}(s-1)} & =\mathrm{D}^{\alpha_{s}} L_{\alpha(s) \dot{\alpha}(s-1)}+\overline{\mathrm{D}}^{\dot{\alpha}_{s}} \bar{L}_{\alpha(s-1) \dot{\alpha}(s)} .
\end{aligned}
$$

Up to redefinitions, these transformations match exactly the ones in [3] and therefore will lead to the construction of the same superspace action.

\section{Discussion}

To summarize our results, we have shown that under the assumption of the natural requirments of ( $i$ ) Supersymmetry [equality of bosonic and fermionic degrees of freedom] and (ii) Superspace [all fields must be generated out of superfields], the problem of off-shell completion of higher spin supermultiplets [finding the list of required supersymmetric auxiliary fields starting from the on-shell data] can be reduced to a set of Diophantine equations. An top of that if we assume (iii) Economy [having no more than what is required], we get a handful of solutions that correspond to the off-shell spectrum of known irreducible higher spin supermultiplets. This new approach provides:

1. a method of classifying all irreducible formulations of a free, massless, arbitrary spin supersymmetric theory, ${ }^{16}$

2. a very natural explanation for why some supermultiplets have more than one formulations (e.g. the matter-gravitino, supergravity and half-integer superspin supermultiplets) and others do not (integer spin supermultiplet),

3. a methodology which gives the explicit off-shell component spectrum for a supermultiplet without knowing the action. The superspace action and the superspace gauge transformation laws can easily be constructed as a direct by-product of this analysis.

Furthermore, for any free, massless, $4 D, \mathcal{N}=1$ theory the supersymmetric auxiliary fields are extremely constrained. Our analysis provides a very good understanding for why that is. We have proved that:

$i$. The fermionic auxiliary fields must always come in pairs $\left(\beta_{\ldots}, \rho_{\ldots}\right)$ and they appear in the action through algebraic terms of the form $\beta \cdots \rho_{\ldots} .+$ c.c. . Also, they are gauge invariant $\left[\delta_{g} \beta_{\ldots}=\delta_{g} \rho_{\ldots}=0\right]$ and will exist only if in the superspace description of the theory there is a fermionic superfield with engineering dimensions $\frac{1}{2}$.

ii. The bosonic auxiliary fields come in two types called Type (1) and Type (2). Type (1) fields always come in pairs $\left(A_{\alpha \dot{\alpha}}, B_{\alpha \beta}\right)$ of a $(1,1)$-tensor $A_{\alpha \dot{\alpha}}$, with $A_{\alpha \dot{\alpha}}= \pm \bar{A}_{\alpha \dot{\alpha}}$

\footnotetext{
${ }^{16}$ Because of the Supersymmetry requirement, the list of auxiliary fields of a supersymmetric theory must correspond to a solution of the Diophantine equation. Hence a classification of the acceptable solutions is a classification of all the possible formulations of the various supermultiplets.
} 
and a $(2,0)$-tensor $B_{\alpha \beta}$ with $B_{\alpha \beta}=B_{\beta \alpha}$. They appear in the action through terms of the form $B^{\alpha \beta} \partial_{\beta}{ }^{\dot{\alpha}} A_{\alpha \dot{\alpha}}+$ c.c. and they have non-trivial gauge transformations

$$
\delta_{g} A_{\alpha \dot{\alpha}}=\partial_{\alpha \dot{\alpha}} \lambda, \lambda= \pm \bar{\lambda}, \quad \delta_{g} B_{\alpha \beta}=\partial_{(\alpha}^{\dot{\beta}} \ell_{\beta) \dot{\beta}}, \ell_{\beta \dot{\beta}}=\mp \bar{\ell}_{\beta \dot{\beta}}, \ell_{\beta \dot{\beta}} \sim \ell_{\beta \dot{\beta}}+\partial_{\beta \dot{\beta}} \ell, \ell=\mp \bar{\ell}
$$

iii. Type (2) auxiliary fields come in singlets $\left(A_{\ldots}\right)$, are gauge invariant $\left[\delta_{g} A_{\ldots}=0\right]$ and appear in the action through the algebraic terms $A \cdots A_{\ldots}$.

These Type (1) auxiliary fields, because of their specific index structure, appear only in low spin supersymmetric theories $(j \leq 2)$ and they are the reason why for low spins there is a zoo of different formulations of the various supermultiplets. The Diophantine equations generated for these systems $Y=1(7.15)$ and $Y=\frac{3}{2}$ (8.10) illustrate in a very clear way the interplay between the Type (1) and Type (2) fields which allowed more solutions, thus more than one superspace formulations.

On the other hand for higher spin supermultiplets all auxiliary bosons are of Type (2) in agreement with the results [1-3]. Nevertheless, the Diophantine point of view through (9.6) provides a clear picture of how one can balance the bosonic and fermionic auxiliary d.o.f. Now we understand that for half-integer spins one can solve the equation (9.5) in two different ways, using identities $(I)$ and $(I I)$, leading to the two different superspace formulations of $Y=s+\frac{1}{2}$ supermultiplets. In contrast, for integer spins and equation (10.1) only identity $(I I)$ is relevant, hence we get a single solution which corresponds to the one superspace formulation of $Y=s$ supermultiplets.

The results presented in this work depend in a very particular manner of embedding the component fields into supermultiplets. This embedding was provided by our assumption for the existence of an underlying superspace formulation that makes the symmetry manifest. An interesting alternative approach to this embedding is the method of "adinkras" [23] which provide a one dimensional network graph description of supermultiplets. In [24, 25] examples are given for how such an embedding works. The adinkras are being used in order to generate a set of adjacency matrices associated with them which provide the various representations of suppersymmetry. These adjacency matrices satisfy a set of algebraic relations called "Garden Algebra" [26-29]. In [24] it was shown that one can use the Garden algebra in order to find off-shell completions of supersymmetric theories, by generating a system of quadratic equations. The spirit of the work presented here is very similar to that and explores similar if not the same issues, where the role of the Garden algebra and its consequence is played by the set of Diophantine equations.

"Perhaps the topic [of this book] will appear fairly difficult to you because it is not yet familiar knowledge and the understanding of beginners is easily confused by mistakes; but with your inspiration and my teaching it will be easy for you to master, because clear intelligence supported by good lessons is a fast route to knowledge."

- Diophantus 


\section{Acknowledgments}

This work was partially supported by the National Science Foundation grant PHY-1315155. This research was also supported in part by CSPT. The work of K. K. was supported by the grant P201/12/G028 of the Grant agency of Czech Republic. K. K. acknowledges the warm hospitality of the Physics departmens of the University of Maryland and Brown University. S. J. G. acknowledges the generous support of the Provostial Visiting Professorship Program and the Department of Physics at Brown University for the very congenial and generous hospitality during the period of this work.

Open Access. This article is distributed under the terms of the Creative Commons Attribution License (CC-BY 4.0), which permits any use, distribution and reproduction in any medium, provided the original author(s) and source are credited.

\section{References}

[1] S.M. Kuzenko, A.G. Sibiryakov and V.V. Postnikov, Massless gauge superfields of higher half integer superspins, JETP Lett. 57 (1993) 534 [INSPIRE].

[2] S.M. Kuzenko and A.G. Sibiryakov, Massless gauge superfields of higher integer superspins, JETP Lett. 57 (1993) 539 [inSPIRE].

[3] S.J. Gates Jr. and K. Koutrolikos, On $4 D, \mathcal{N}=1$ massless gauge superfields of arbitrary superhelicity, JHEP 06 (2014) 098 [arXiv:1310.7385] [arXiv:1310.7386] [INSPIRE].

[4] S.J. Gates Jr. and K. Koutrolikos, A dynamical theory for linearized massive superspin 3/2, JHEP 03 (2014) 030 [arXiv: 1310.7387] [INSPIRE].

[5] S.J. Gates Jr., S.M. Kuzenko and G. Tartaglino-Mazzucchelli, New massive supergravity multiplets, JHEP 02 (2007) 052 [hep-th/0610333] [INSPIRE].

[6] C. Fronsdal, Massless Fields with Integer Spin, Phys. Rev. D 18 (1978) 3624 [inSPIRE].

[7] J. Fang and C. Fronsdal, Massless Fields with Half Integral Spin, Phys. Rev. D 18 (1978) 3630 [INSPIRE].

[8] L.P.S. Singh and C.R. Hagen, Lagrangian formulation for arbitrary spin. 2. The fermion case, Phys. Rev. D 9 (1974) 910 [inSPIRE].

[9] L.P.S. Singh and C.R. Hagen, Lagrangian formulation for arbitrary spin. 1. The boson case, Phys. Rev. D 9 (1974) 898 [INSPIRE].

[10] P.S. Howe, K.S. Stelle and P.K. Townsend, The Relaxed Hypermultiplet: An Unconstrained $N=2$ Superfield Theory, Nucl. Phys. B 214 (1983) 519 [InSPIRE].

[11] C.F. Doran, M.G. Faux, S.J. Gates Jr., T. Hubsch, K.M. Iga and G.D. Landweber, On the matter of $N=2$ matter, Phys. Lett. B 659 (2008) 441 [arXiv:0710.5245] [INSPIRE].

[12] M. Faux, The Conformal Hyperplet, Int. J. Mod. Phys. A 32 (2017) 1750079 [arXiv: 1610.07822] [INSPIRE].

[13] B.B. Deo and S.J. Gates Jr., Comments on nonminimal $N=1$ scalar multiplets, Nucl. Phys. B 254 (1985) 187 [INSPIRE].

[14] W. Siegel, Manifest Lorentz Invariance Sometimes Requires Nonlinearity, Nucl. Phys. B 238 (1984) 307 [INSPIRE]. 
[15] V. Ogievetsky and E. Sokatchev, On Vector Superfield Generated by Supercurrent, Nucl. Phys. B 124 (1977) 309 [INSPIRE].

[16] V.I. Ogievetsky and E.S. Sokatchev, The Axial Superfield and the Supergravity Group, Yad. Fiz. 28 (1978) 1631 [INSPIRE].

[17] W. Siegel and S.J. Gates Jr., Superfield Supergravity, Nucl. Phys. B 147 (1979) 77 [inSPIRE].

[18] B. de Wit and J.W. van Holten, Multiplets of Linearized SO(2) Supergravity, Nucl. Phys. B 155 (1979) 530 [INSPIRE].

[19] S.J. Gates Jr. and W. Siegel, $(3 / 2,1)$ Superfield of O(2) Supergravity, Nucl. Phys. B 164 (1980) 484 [INSPIRE].

[20] S. Deser, P.K. Townsend and W. Siegel, Higher Rank Representations of Lower Spin, Nucl. Phys. B 184 (1981) 333 [INSPIRE].

[21] S.J. Gates Jr. and W. Siegel, Variant superfield representations, Nucl. Phys. B 187 (1981) 389 [INSPIRE].

[22] V.I. Ogievetsky and E. Sokatchev, On Gauge Spinor Superfield, JETP Lett. 23 (1976) 58 [INSPIRE].

[23] M. Faux and S.J. Gates Jr., Adinkras: A Graphical technology for supersymmetric representation theory, Phys. Rev. D 71 (2005) 065002 [hep-th/0408004] [INSPIRE].

[24] M. Calkins, D.E.A. Gates, S.J. Gates Jr. and W.M. Golding, Think Different: Applying the Old Macintosh Mantra to the Computability of the SUSY Auxiliary Field Problem, JHEP 04 (2015) 056 [arXiv : 1502.04164] [INSPIRE].

[25] M. Calkins, D.E.A. Gates, S.J. Gates and B. McPeak, Is it possible to embed a $4 D, \mathcal{N}=4$ supersymmetric vector multiplet within a completely off-shell adinkra hologram?, JHEP 05 (2014) 057 [arXiv: 1402.5765] [inSPIRE].

[26] C.F. Doran, M.G. Faux, S.J. Gates Jr., T. Hübsch, K.M. Iga and G.D. Landweber, On graph-theoretic identifications of Adinkras, supersymmetry representations and superfields, Int. J. Mod. Phys. A 22 (2007) 869 [math-ph/0512016] [inSPIRE].

[27] C.F. Doran, M.G. Faux, S.J. Gates Jr., T. Hübsch, K.M. Iga and G.D. Landweber, Relating Doubly-Even Error-Correcting Codes, Graphs and Irreducible Representations of N-Extended Supersymmetry, arXiv:0806.0051 [INSPIRE].

[28] C.F. Doran et al., Codes and Supersymmetry in One Dimension, Adv. Theor. Math. Phys. 15 (2011) 1909 [arXiv:1108.4124] [INSPIRE].

[29] C.F. Doran et al., Topology Types of Adinkras and the Corresponding Representations of $N$-Extended Supersymmetry, arXiv:0806.0050 [INSPIRE]. 